\title{
NOVIKOV HOMOLOGY, TWISTED ALEXANDER POLYNOMIALS, AND THURSTON CONES
}

\author{
A. V. PAJITNOV
}

\begin{abstract}
Let $M$ be a connected CW complex, and let $G$ denote the fundamental group of $M$. Let $\pi$ be an epimorphism of $G$ onto a free finitely generated Abelian group $H$, let $\xi: H \rightarrow \mathbf{R}$ be a homomorphism, and let $\rho$ be an antihomomorphism of $G$ to the group GL( $V)$ of automorphisms of a free finitely generated $R$-module $V$ (where $R$ is a commutative factorial ring).

To these data, we associate the twisted Novikov homology of $M$, which is a module over the Novikov completion of the ring $\Lambda=R[H]$. The twisted Novikov homology provides the lower bounds for the number of zeros of any Morse form whose cohomology class equals $\xi \circ \pi$. This generalizes a result by H. Goda and the author.

In the case when $M$ is a compact connected 3-manifold with zero Euler characteristic, we obtain a criterion for the vanishing of the twisted Novikov homology of $M$ in terms of the corresponding twisted Alexander polynomial of the group $G$.

We discuss the relationship of the twisted Novikov homology with the Thurston norm on the 1-cohomology of $M$.

The electronic preprint of this work (2004) is available from the ArXiv.
\end{abstract}

\section{$\S 1$. INTRODUCTION}

Let $M$ be a closed manifold, $f: M \rightarrow S^{1}$ a circle-valued Morse function on $M$. Let $m_{k}(f)$ denote the number of critical points of $f$ of index $k$. The Morse-Novikov theory provides lower bounds for the numbers $m_{k}(f)$ that are computable in terms of the homotopy type of $M$ and the homotopy class of $f$. The general method of obtaining such bounds is outlined as follows (see [19, 6, 21, 23]). Consider a regular covering $\mathcal{P}: \bar{M} \rightarrow M$ with structure group $G$ such that the function $f \circ \mathcal{P}: \bar{M} \rightarrow S^{1}$ is homotopic to zero (or equivalently, $f$ lifts to a Morse function $\bar{M} \rightarrow \mathbb{R}$ ). The induced homomorphism $f_{*}: \pi_{1}(M) \rightarrow \mathbb{Z}$ can be factored through a homomorphism $\xi=\xi(f): G \rightarrow \mathbb{Z}$.

Applying the standard method of counting gradient flow lines (see [39]), one obtains a chain complex $\mathcal{N}_{*}$ (the Novikov complex) over a certain completion $\widehat{\mathcal{L}}_{\xi}$ of the group $\operatorname{ring} \mathcal{L}=\mathbb{Z}[G] .{ }^{1}$ The Novikov complex is freely generated over $\widehat{\mathcal{L}}_{\xi}$ by the critical points of $\omega$, and its homology is isomorphic to the homology of the tensor product

$$
\widehat{\mathcal{C}}_{*}(\bar{M}, \xi)=\widehat{\mathcal{L}}_{\xi} \otimes_{\mathcal{L}} \mathcal{C}_{*}(\bar{M}),
$$

where $\mathcal{C}_{*}(\bar{M})$ is the cellular chain complex of the covering $\bar{M}$. In particular, if the chain complex (1) is not contractible, the function $f$ must have at least one critical point. Developing this observation further, one can obtain lower bounds for the numbers $m_{k}(f)$ in terms of the numerical invariants of the homology of the chain complex (1).

Let us discuss different possible choices of the covering $\mathcal{P}$. Certainly, the universal covering $\widetilde{M} \rightarrow M$ contains the maximum amount of information. The disadvantage is that the corresponding ring $\widehat{\mathcal{L}}_{\xi}$, being a completion of the group ring of the fundamental

2000 Mathematics Subject Classification. Primary 57Rxx.

${ }^{1}$ See the definition of $\widehat{\mathcal{L}}_{\xi}$ in Subsection 2.1 . 
group, may be very complicated, and it can be difficult to extract the necessary numerical data.

Another obvious possibility is the infinite cyclic covering

$$
\mathcal{P}_{f}: \bar{M}_{f} \rightarrow M
$$

induced by $f$ from the universal covering $\mathbb{R} \rightarrow S^{1}$. Here the ring $\widehat{\mathcal{L}}_{\xi}$ is a principal ideal domain, and the explicit lower bounds for the numbers of the critical points are easy to deduce (see Subsection 2.1 for more details).

An intermediate choice is the maximal free abelian covering

$$
\mathcal{P}_{a b}: \bar{M}_{a b} \rightarrow M \text {. }
$$

The structure group of this covering is equal to $H_{1}(M) /$ Tors. In this case, the Novikov ring is a completion of the Laurent polynomial ring in several variables, and, in general, its homology properties are rather complicated. But this choice has an advantage that for any function $f: M \rightarrow S^{1}$ the function $f \circ \mathcal{P}_{a b}$ is homotopic to zero. This allows us to study the dependence of the Novikov homology on the class

$$
\xi=\xi(f) \in \operatorname{Hom}\left(H_{1}(M) / \operatorname{Tors}, \mathbb{Z}\right) \approx H^{1}(M, \mathbb{Z}) .
$$

In particular, one can get some information about the set of all $\xi$ such that the Novikov homology

$$
\widehat{H}_{*}^{a b}(M, \xi)=H_{*}\left(\widehat{\mathcal{C}}_{*}\left(\bar{M}_{a b}, \xi\right)\right)
$$

vanishes and, therefore, obtain information about the set of classes in $H^{1}(M, \mathbb{Z})=\left[M, S^{1}\right]$ representable by fibrations.

Definition 1.1. Let $H$ be a finitely generated free Abelian group. Put

$$
H_{\mathbb{R}}=H \otimes \mathbb{R}, \quad H_{\mathbb{R}}^{\prime}=\operatorname{Hom}(H, \mathbb{R})=\operatorname{Hom}(H, \mathbb{Z}) \otimes \mathbb{R} .
$$

A closed cone in $H_{\mathbb{R}}^{\prime}$ is a closed subset $C$ such that $v \in C \Rightarrow \lambda \cdot v \in C$ for every $\lambda \geq 0$.

An open cone in $H_{\mathbb{R}}^{\prime}$ is an open subset $D$ such that $v \in D \Rightarrow \lambda \cdot v \in D$ for every $\lambda>0$.

An integral hyperplane of the vector space $H_{\mathbb{R}}^{\prime}=\operatorname{Hom}(H, \mathbb{R})$ is a vector subspace of codimension 1 in $H_{\mathbb{R}}^{\prime}$ having a basis formed by elements of $\operatorname{Hom}(H, \mathbb{Z})$.

A connected component of the complement to a given hyperplane $\Gamma$ will be called the open half-space corresponding to $\Gamma$. The closure of an open half-space will be called the closed half-space corresponding to $\Gamma$.

A closed cone that is the intersection of a finite family of closed half-spaces corresponding to integral hyperplanes is called a closed polyhedral cone.

An open cone that is the intersection of a finite family of open half-spaces corresponding to integral hyperplanes is called an open polyhedral cone.

A subset $C \subset H_{\mathbb{R}}^{\prime}$ is called an open polyhedral conical subset if it is empty, or equals $H_{\mathbb{R}}^{\prime} \backslash\{0\}$, or is a finite disjoint union of open polyhedral cones.

A subset $A \subset H_{\mathbb{R}}^{\prime}$ is called a quasipolyhedral conical subset if there is an open polyhedral conical subset $C$ and a finite union $D$ of integral hyperplanes such that $D \cup C=$ $D \cup A$.

The next theorem follows from the main theorem of my paper [22], it is based on earlier results of J.-ClSikorav (see [21]).

Theorem 1.2. The set of all classes $\xi \in H^{1}(M, \mathbb{Z})$ such that the Novikov homology $\widehat{H}_{*}^{a b}(M, \xi)$ vanishes is the intersection with $H^{1}(M, \mathbb{Z})$ of a quasi-polyhedral conical subset of $H^{1}(M, \mathbb{R}) .^{2}$

\footnotetext{
${ }^{2}$ The results of [21] and [22] pertain actually to a more general case of arbitrary homomorphisms $\pi_{1}(M) \rightarrow \mathbb{R}$, and not only homomorphisms $\pi_{1}(M) \rightarrow \mathbb{Z}$; see the discussion below.
} 
Now we proceed to non-Abelian coverings. In the joint work with Goda [11], we introduced a new version of the Novikov homology. We call it the twisted Novikov homology. The input data for the construction is: a connected CW complex $M$, a homomorphism $\xi: \pi_{1}(M) \rightarrow \mathbb{Z}$, and an anti-homomorphism $\rho: \pi_{1}(M) \rightarrow \operatorname{GL}(n, \mathbb{Z})$. The resulting twisted Novikov homology groups are modules over the principal ideal domain $\mathbb{Z}((t))$, so the numerical invariants are easily extracted from the homological data. On the other hand, the non-Abelian homological algebra of the universal covering of $M$ is encoded in this homology via the representation $\rho$. The construction of the twisted Novikov homology is motivated by the notion of the twisted Alexander polynomial for knots and links. (See the papers [15] of Lin and [37] of M. Wada for the definition and properties of the twisted Alexander polynomials, and the paper [9] of Goda, T. Kitano, T. Morifuji for applications of twisted Alexander polynomials to fibering obstructions for knots and links.)

The definition of the twisted Novikov homology generalizes immediately to the case of arbitrary cohomology classes $\xi \in H^{1}(M, \mathbb{R})$. The input data for this construction is as follows. Let $R$ be a commutative ring. Let $V$ be a finitely generated free left $R$-module. Denote by $\mathrm{GL}_{R}(V)$ the group of all automorphisms of $V$ over $R$. Let $\rho: G \rightarrow \mathrm{GL}_{R}(V)$ be an antihomomorphism (that is, $\rho(a b)=\rho(b) \rho(a)$ for all $a, b \in G ; \rho$ will also be called a right representation). Let $\pi: G \rightarrow H$ be an epimorphism of $G$ onto a free finitely generated Abelian group $H$. Let $\xi: H \rightarrow \mathbb{R}$ be a group homomorphism.

To this data array, we associate the twisted Novikov homology as follows. Let $\Lambda=$ $R[H]$; put $V^{H}=\Lambda \otimes_{R} V$; define a right representation $\rho_{\pi}: G \rightarrow \mathrm{GL}_{\Lambda}\left(V^{H}\right)$ by the formula

$$
\rho_{\pi}(g)\left(\sum_{i} \lambda_{i} \otimes v_{i}\right)=\sum_{i}\left(\pi(g) \lambda_{i}\right) \otimes \rho(g) v_{i}
$$

Form the tensor product of the cellular chain complex $\mathcal{C}_{*}(\widetilde{M})$ of the universal covering with the right $\mathbb{Z} G$-module $V^{H}$ :

$$
\widetilde{\mathcal{C}}_{*}\left(M, \rho_{\pi}\right)=V^{H} \otimes_{\mathbb{Z} G} \mathcal{C}_{*}(\widetilde{M}) .
$$

This is a chain complex of left free $\Lambda$-modules (observe that

$$
\operatorname{rk}_{\Lambda}\left(\widetilde{\mathcal{C}}_{k}\left(M, \rho_{\pi}\right)\right)=n \cdot \operatorname{rk} \mathcal{C}_{k}(\widetilde{M}),
$$

where $n$ is the rank of $V$ over $R$ ). Apply the tensor product with $\widehat{\Lambda}_{\xi}$ to obtain the chain complex

$$
\widehat{\mathcal{C}}_{*}\left(M, \rho_{\pi}, \xi\right)=\widehat{\Lambda}_{\xi} \otimes_{\Lambda} \widetilde{\mathcal{C}}_{*}\left(M, \rho_{\pi}\right) .
$$

Its homology

$$
\widehat{H}_{*}\left(M, \rho_{\pi}, \xi\right)=H_{*}\left(\widehat{\mathcal{C}}_{*}\left(M, \rho_{\pi}, \xi\right)\right)
$$

is called the twisted Novikov homology. The twisted Novikov homology of our paper [11] corresponds to the particular case when $H=\mathbb{Z}$, and the homomorphism $\xi: H \rightarrow \mathbb{R}$ above is the inclusion $\mathbb{Z} \hookrightarrow \mathbb{R}$.

The present generalization simplifies the statements of several theorems below. It also has a geometrical background; it corresponds to Morse forms, while the framework of our paper [11] was related to circle-valued Morse functions. (Recall that a closed 1-form on a manifold $M$ is called a Morse form if locally it is a differential of a Morse function.) Namely, we have the following theorem.

Theorem 1.3. Let $\omega$ be a Morse form on a closed connected manifold $M$. Assume that the cohomology class $[\omega] \in H^{1}(M, \mathbb{R})=\operatorname{Hom}\left(\pi_{1}(M), \mathbb{R}\right)$ can be factored as $[\omega]=\xi \circ \pi$, 
where $\pi: \pi_{1}(M) \rightarrow H$ is an epimorphism onto a free Abelian group, and $\xi: H \rightarrow \mathbb{R}$ is a homomorphism. Then there is a chain complex $\mathcal{N}_{*}^{\rho}$ such that

1) $\mathcal{N}_{k}^{\rho}$ is a free $\widehat{\Lambda}_{\xi}$-module with $n \cdot m_{k}(\omega)$ free generators in degree $k$ (where $m_{k}(\omega)$ stands for the number of zeros of the form $\omega$ of index $k$ );

2) $\mathcal{N}_{*}^{\rho}$ is chain homotopy equivalent to $\widehat{\mathcal{C}_{*}}\left(\widetilde{M}, \rho_{\pi}, \xi\right)$.

In particular, if the cohomology class $[\omega]$ contains a nowhere vanishing 1-form, then the twisted Novikov homology $\widehat{H}_{*}\left(M, \rho_{\pi}, \xi\right)$ equals zero.

The most natural choice of the epimorphism $\pi: \pi_{1}(M) \rightarrow H$ is the projection $\pi_{1}(M) \rightarrow H_{1}(M) /$ tors onto the integral homology group of $M$ modulo its torsion subgroup. The corresponding twisted Novikov homology will be denoted by $\widehat{H}_{*}(M, \rho, \xi)$.

Definition 1.4. A nonzero homomorphism

$$
\xi \in \operatorname{Hom}\left(H_{1}(M), \mathbb{R}\right)=\operatorname{Hom}\left(H_{1}(M) / \operatorname{Tors}, \mathbb{R}\right)=H^{1}(M, \mathbb{R})
$$

is called $\rho$-acyclic if the $\rho$-twisted Novikov homology $\widehat{H}_{*}(M, \rho, \xi)$ vanishes. The set of all $\rho$-acyclic classes will be denoted by $\mathcal{V}_{\text {alg }}(M, \rho)$.

The reason for studying the $\rho$-acyclic classes is that any class $\xi$ containing a nowhere vanishing closed 1 -form is $\rho$-acyclic for any representation $\rho$. An immediate generalization of Theorem 1.2 leads to the following result about the algebraic structure of the set of $\rho$-acyclic classes.

Theorem 1.5. For a given right representation $\rho$ of $\pi_{1}(M)$, the set $\mathcal{V}_{\text {alg }}(M, \rho)$ of all $\rho$-acyclic classes $\xi$ is a quasipolyhedral conical subset.

In general we do not know whether the set of all $\rho$-acyclic classes is an open polyhedral conical subset. In other words, we can describe the structure of the set of all $\rho$-acyclic classes only up to some finite union of hyperplanes in $H^{1}(M, \mathbb{R})$. However, in the case when $M$ is a 3 -manifold, we have a much stronger assertion, which is the main result of the present paper (see $\S 5$ ).

Theorem 1.6. Let $M$ be a connected compact three-dimensional manifold (maybe with nonempty boundary) such that $\chi(M)=0$. Let $\rho$ be a right representation of $\pi_{1}(M)$. The following statements are true.

1. The set $\mathcal{V}_{\text {alg }}(M, \rho)$ of all $\rho$-acyclic classes $\xi$ is an open polyhedral conical subset.

2. This subset is entirely determined by the twisted Alexander polynomial associated with the group $\pi_{1}(M)$ and the representation $\rho$.

The open polyhedral cones forming the subset $\mathcal{V}_{\text {alg }}(M, \rho)$ will be called $\rho$-acyclicity cones. The theorem above implies that the set of all $\rho$-acyclic classes depends only on the group $\pi_{1}(M)$ and the representation $\rho$.

Along with the twisted Alexander polynomials, the proof of the above theorem involves additional polynomial invariants of the chain complexes, which we introduce in $\S 3$ and call the Fitting invariants. These invariants are defined as the GCDs of the minors of the second boundary operator of the chain complex (3). We show that they are directly related to the Novikov homology, and in many cases the Novikov homology in degree one can be computed from the sequence of the Fitting invariants. The twisted Alexander polynomial is a much more sophisticated invariant, but it turns out that the image of the twisted Alexander polynomial in the Novikov completion $\widehat{\Lambda}_{\xi}$ is essentially the image of the corresponding Fitting invariant.

Theorem 1.6 is related to Thurston's famous theorem [34], which implies that the set $\mathcal{V}(M)$ of all classes $\xi \in H^{1}(M, \mathbb{R})$ representable by closed nowhere vanishing 1 -forms is a finite union of open polyhedral cones, namely the cones on certain faces of the unit 
ball of the Thurston norm on $H^{1}(M, \mathbb{R})$. We shall call these cones Thurston cones. For every right representation $\rho$ of $\pi_{1}(M)$ in $\operatorname{GL}\left(\mathbb{Z}^{n}\right)$, the set $\mathcal{V}(M)$ is contained in the set $\mathcal{V}_{\text {alg }}(M, \rho)$ of all $\rho$-acyclic classes:

$$
\mathcal{V}(M) \subset \mathcal{V}_{\text {alg }}(M, \rho)
$$

so each Thurston cone is contained in a $\rho$-acyclicity cone. The $\rho$-acyclicity cones are computable in terms of the twisted Alexander polynomial, which is in its turn computable in terms of the Alexander matrix associated with any finite presentation of the group $\pi_{1}(M)$. Thus, in a sense, the set $\mathcal{V}_{\text {alg }}(M, \rho)$ is a computable upper bound for the set $\mathcal{V}(M)$. Let

$$
\mathcal{V}_{\text {alg }}(M)=\bigcap_{\rho} \mathcal{V}_{\text {alg }}(M, \rho)
$$

where $\rho$ ranges over the set of all right representations $\rho$ of $\pi_{1}(M)$ in $\operatorname{GL}\left(\mathbb{Z}^{n}\right)$. Then we have

$$
\mathcal{V}(M) \subset \mathcal{V}_{\text {alg }}(M)
$$

It is an interesting problem to investigate the relationship between the two sets, and in particular answer the following question:

Question. For which manifolds does the identity $\mathcal{V}(M)=\mathcal{V}_{\text {alg }}(M)$ hold true?

We do not know examples of manifolds for which $\mathcal{V}(M) \neq \mathcal{V}_{\text {alg }}(M)$. On the other hand, it is easy to construct manifolds for which

$$
\mathcal{V}(M) \neq \bigcap_{\rho \in \mathcal{R}_{f}} \mathcal{V}_{\mathrm{alg}}(M, \rho),
$$

where $\mathcal{R}_{f}$ is the set of all right representations of $G$ over finite fields (see Subsection 5.3). For such manifolds, the right representations over finite fields are not sufficient to detect all the cohomology classes representable by nonsingular 1-forms.

A certain amount of computation will be necessary to clarify the relationship between $\mathcal{V}(M)$ and $\mathcal{V}_{\text {alg }}(M)$ and answer the question above. The recent progress in the software related to the computations of invariants of knots and links, especially Kodama's KNOT program, allows us to hope that such computations can be carried out.

Electronic version. The e-print of the present paper (2004) is available at the ArXiv (math.GT/0406498).

Notes on the terminology. Ring means an associative ring with a unit. For a ring $R$, we denote by $R^{\bullet}$ the multiplicative group of all invertible elements of $R$. Module always means a left module if the contrary is not stated explicitly. The homology of a space $X$ with integral coefficients will be denoted by $H_{*}(X)$. For a left module $V$ over a commutative ring $R$, we denote by $\mathrm{GL}_{R}(V)$ the group of all $R$-automorphisms of $V$. When the ring $R$ is clear from the context, we use the abbreviated notation $\operatorname{GL}(V)$. For a ring $R$, the symbol $\mathrm{GL}(n, R)$ denotes the multiplicative group of all invertible $(n \times n)$-matrices with coefficients in $R$.

\section{§2. Twisted Novikov homology}

\subsection{Novikov homology.}

Definition 2.1. Let $G$ be a group, and let $R$ be a commutative ring with a unit. Put $\mathcal{L}=R[G]$. Let $\xi: G \rightarrow \mathbb{R}$ be a group homomorphism. Let $\widehat{\widehat{\mathcal{L}}}$ be the set of all formal 
linear combinations (infinite in general) of the form $\lambda=\sum_{g \in G} n_{g} g$, where $n_{g} \in R$. For $\lambda \in \widehat{\widehat{\mathcal{L}}}$ and $C \in \mathbb{R}$, put

$$
\sup (\lambda, C)=\left\{g \in G \mid n_{g} \neq 0, \xi(g)>C\right\} .
$$

The Novikov ring is defined as follows:

$$
\widehat{\mathcal{L}}_{\xi}=\{\lambda \in \widehat{\widehat{\mathcal{L}}} \mid \sup (\lambda, C) \text { is finite for every } C \in \mathbb{R}\} .
$$

(It is easy to show that the subset $\widehat{\mathcal{L}}_{\xi} \subset \widehat{\widehat{\mathcal{L}}}$ has indeed a natural structure of a ring containing $\mathcal{L}$ as a subring.)

Example 2.2. Let $G$ be an Abelian finitely generated group. A homomorphism $\xi$ : $G \rightarrow \mathbb{R}$ is called rational if $\xi=\lambda \cdot \xi_{0}$, where $\xi_{0}: G \rightarrow \mathbb{Z}$ is a homomorphism and $\lambda \in \mathbb{R}$. Equivalently, $\xi$ is rational if $\xi=0$ or $\xi(G) \approx \mathbb{Z}$. When $\xi$ is nonzero and rational, we have an isomorphism

$$
\widehat{\mathcal{L}}_{\xi} \approx K[[t]]\left[t^{-1}\right], \quad \text { where } K=R[\operatorname{Ker} \xi],
$$

so that the ring $\widehat{\mathcal{L}}_{\xi}$ is a localization of the ring $K[[t]]$ of power series. In particular, $\widehat{\mathcal{L}}_{\xi}$ is Noetherian.

Example 2.3. Let $G$ be a free Abelian finitely generated group. In this case, $\mathcal{L}=R[G]$ is isomorphic to the ring of Laurent polynomials in $k$ variables (where $k=\operatorname{rk} G$ ) with coefficients in $R$.

When $\xi: G \rightarrow \mathbb{R}$ is injective (such homomorphisms are also called totally irrational), the algebraic properties of the ring $\widehat{\mathcal{L}}_{\xi}$ are surprisingly simple.

Theorem 2.4. If $\xi$ is totally irrational and $R$ is a principal ideal domain, then the ring $\widehat{\mathcal{L}}_{\xi}$ is also a principal ideal domain.

For the case where $R=\mathbb{Z}$, this theorem is due to J.-Cl. Sikorav (his proof was published in [21]). The proof in the general case is similar. Observe that if $R$ is a field, then $\widehat{\mathcal{L}}_{\xi}$ is also a field.

The main topological applications of these constructions are in the theory of Morse forms. Let $M$ be a closed connected $C^{\infty}$ manifold, and let $\omega$ be a Morse form on $M$. Let $[\omega] \in H^{1}(M, \mathbb{R})$ denote the de Rham cohomology class of $\omega$; then $[\omega]$ can also be identified with a homomorphism $G=\pi_{1}(M) \rightarrow \mathbb{R}$. Let $\widehat{\mathfrak{L}}_{[\omega]}$ denote the corresponding Novikov completion of the ring $\mathfrak{L}=\mathbb{Z} G$. The next theorem relates the homotopy type of the completed chain complex

$$
\widehat{\mathcal{C}}_{*}(\widetilde{M},[\omega])=\widehat{\mathfrak{L}}_{[\omega]} \otimes_{\mathfrak{L}} \mathcal{C}_{*}(\widetilde{M})
$$

(where $\mathcal{C}_{*}(\widetilde{M})$ denotes the cellular chain complex of the universal covering of $M$ ) and the geometrical properties of the form $\omega$. Denote by $S_{k}(\omega)$ the set of zeros of $\omega$ of index $k$, and by $S(\omega)$ the set of all zeros.

Theorem 2.5. There is a chain complex $\mathcal{N}_{*}$ over the ring $\widehat{\mathfrak{L}}_{[\omega]}$ such that:

1) $\mathcal{N}_{k}$ is freely generated over $\widehat{\mathfrak{L}}_{[\omega]}$ by $S_{k}(\omega)$;

2) the chain complexes $\mathcal{N}_{*}$ and $\widehat{\mathcal{C}}_{*}(M,[\omega])$ are chain homotopy equivalent.

For the case of integral classes $[\omega] \in H^{1}(M, \mathbb{Z})$, the theorem follows from the existence of the Novikov complex $\mathcal{N}_{*}(f, v)$ associated with a circle-valued Morse map $f$ such that $d f=\omega$ and any transverse $f$-gradient $v$ (see [23]). The case of rational classes $[\omega] \in$ $H^{1}(M, \mathbb{Q})$ follows immediately. For the case of 1 -forms belonging to arbitrary cohomology classes, see the later paper by Latour [14] and also the papers [30, 31] of Schütz. 
2.2. Twisted Novikov homology. In this subsection, we begin our study of the twisted Novikov homology. Let $M$ be a connected CW complex. Let $\rho: G \rightarrow \mathrm{GL}_{R}(V)$ be any right representation, $\pi: G \rightarrow H$ an epimorphism, and $\xi: H \rightarrow \mathbb{R}$ a homomorphism. In the introduction, with these data we associated a chain complex $\widehat{\mathcal{C}}_{*}\left(M, \rho_{\pi}, \xi\right)$ over $\widehat{\Lambda}_{\xi}$ (where $\Lambda=R[H]$ ). When $\xi: H \rightarrow \mathbb{R}$ is a monomorphism and $R$ is a principal ideal domain, the ring $\widehat{\Lambda}_{\xi}$ is also a principal ideal domain. In this case, put

$$
\begin{aligned}
& \widehat{b}_{i}\left(M, \rho_{\pi}, \xi\right)=\operatorname{rk}_{\widehat{\Lambda}_{\xi}} H_{i}\left(\widehat{\mathcal{C}}_{*}\left(M ; \rho_{\pi}, \xi\right)\right), \\
& \widehat{q}_{i}\left(M, \rho_{\pi}, \xi\right)=\operatorname{t.n} \widehat{\Lambda}_{\xi} H_{i}\left(\widehat{\mathcal{C}}_{*}\left(M ; \rho_{\pi}, \xi\right)\right)
\end{aligned}
$$

(where t.n. stands for the torsion number of the module, i.e., the minimal possible number of generators of its torsion submodule). Observe that if $R$ is a field, then all the numbers $\widehat{q}_{i}\left(M, \rho_{\pi}, \xi\right)$ vanish.

Theorem 2.6. For a given right representation $\rho$ and a given homomorphism $\pi: G \rightarrow$ $H$, the numbers $b_{k}\left(M, \rho_{\pi}, \xi\right)$ do not depend on the monomorphism $\xi$. There is a set $\Gamma \subset H_{\mathbb{R}}^{\prime}=\operatorname{Hom}(H, \mathbb{R})$ which is a finite union of integral hyperplanes such that in every connected component of the complement $H_{\mathbb{R}}^{\prime} \backslash \Gamma$ the numbers $\widehat{q}_{k}\left(M, \rho_{\pi}, \xi\right)$ do not depend on $\xi$.

In the particular case of the trivial representation, this theorem is due to Sikorav (see [21]); the proof in the general case is similar.

Corollary 2.7. There is an open polyhedral conical subset $S \subset H_{\mathbb{R}}^{\prime}=\operatorname{Hom}(H, \mathbb{R})$ such that a monomorphism $\xi: H \rightarrow \mathbb{R}$ is in $S$ if and only if the Novikov homology $\widehat{H}_{*}\left(M ; \rho_{\pi}, \xi\right)$ vanishes.

It is natural to ask whether we can drop the condition of injectivity for $\xi$ in the corollary above and still keep the conclusion of the corollary. I do not know if the answer is positive in general, but this is the case when $M$ is a compact connected 3-manifold with $\chi(M)=0$, and $\pi: G \rightarrow H$ is the projection onto $H=H_{1}(M, \mathbb{Z}) /$ Tors (see $\S 5$ ).

Let us proceed to the applications of the twisted Novikov homology in the theory of Morse forms. Now we assume that $M$ is a closed connected $C^{\infty}$ manifold. Let $\omega$ be a Morse form on $M$. The de Rham cohomology class $[\omega] \in H^{1}(M, \mathbb{R})$ of $\omega$ can be identified with a homomorphism $G=\pi_{1}(M) \rightarrow \mathbb{R}$. Let $\pi: G \rightarrow H$ be an epimorphism such that $[\omega]$ factors through $\pi$, so that $[\omega]=\xi \circ \pi$, where $\xi: H \rightarrow \mathbb{R}$ is a homomorphism.

Theorem 2.8. There is a chain complex $\mathcal{N}_{*}^{\rho}$ over the ring $\widehat{\Lambda}_{\xi}$ such that:

1) $\mathcal{N}_{k}^{\rho}$ is a free $\widehat{\Lambda}_{\xi}$-module with $n \cdot m_{k}(\omega)$ free generators;

2) the $\widehat{\Lambda}_{\xi}$-modules $H_{*}\left(\mathcal{N}_{*}^{\rho}\right)$ and $\widehat{H}_{*}\left(M ; \rho_{\pi}, \xi\right)$ are isomorphic.

Proof. Let $\widehat{V}^{H}=\widehat{\Lambda}_{\xi} \otimes_{\Lambda} V^{H}$; the composition of the right representation $\rho_{\pi}$ with the natural inclusion $\mathrm{GL}_{\Lambda}\left(V^{H}\right) \hookrightarrow \mathrm{GL}_{\widehat{\Lambda}_{\xi}}\left(\widehat{V}^{H}\right)$ determines a right representation $\widehat{\rho}_{\pi}$ of $G$ in $\mathrm{GL}_{\widehat{\Lambda}_{\xi}}\left(\widehat{V}^{H}\right)$. By using the factorization $[\omega]=\xi \circ \pi$, it is not difficult to check that the representation $\widehat{\rho}_{\pi}$ extends to a structure of a right $\widehat{\mathfrak{L}}_{[\omega]}$-module on $\widehat{V}^{H}$, and we have

$$
\widehat{\mathcal{C}}_{*}\left(M ; \rho_{\pi}, \xi\right)=\widehat{V}^{H} \otimes_{\widehat{\mathfrak{L}}_{[\omega]}} \widehat{\mathcal{C}}_{*}(\widetilde{M},[\omega]) .
$$

Now our theorem immediately follows from Theorem 2.5.

Corollary 2.9. Let $\omega$ be a closed 1-form without zeros. Assume that

$$
[\omega]=\xi \circ \pi \in \operatorname{Hom}(G, \mathbb{R})=H^{1}(M, \mathbb{R}),
$$

where $\xi: H \rightarrow \mathbb{R}$ is a homomorphism and $\pi: G \rightarrow H$ is an epimorphism. Then $\widehat{H}_{*}\left(M ; \rho_{\pi}, \xi\right)=0$. 
Corollary 2.10. Let $\omega$ be a Morse form. Assume that

$$
[\omega]=\xi \circ \pi \in \operatorname{Hom}(G, \mathbb{R})=H^{1}(M, \mathbb{R}),
$$

where $\xi: H \rightarrow \mathbb{R}$ is a monomorphism and $\pi: G \rightarrow H$ is an epimorphism. Assume that $R$ is a principal ideal domain. Then

$$
m_{i}(\omega) \geq \widehat{b}_{i}\left(M, \rho_{\pi}, \xi\right)+\widehat{q}_{i}\left(M, \rho_{\pi}, \xi\right)+\widehat{q}_{i-1}\left(M, \rho_{\pi}, \xi\right) .
$$

\section{§3. Fitting invariants of Chain COMPlexes}

Let $A$ be a finitely generated module over a commutative $\operatorname{ring} Q$. Let

$$
0 \longleftarrow A \longleftarrow C_{0} \stackrel{\lambda}{\longleftarrow} C_{1}
$$

be a presentation for $A$ with free finitely generated modules $C_{0}$ and $C_{1}$. By definition, the $k$ th Fitting ideal of $A$ is the ideal generated by all $(n-k) \times(n-k)$-minors of the matrix of the homomorphism $\lambda$, where $n=\operatorname{rk} C_{0}$ (see $\left.[5, \S 20.2]\right)$.

In this section, we give a generalization of this construction and, for every chain complex $C_{*}$ of free finitely generated $Q$-modules, we define a family of ideals of $Q$ (the Fitting ideals of $C_{*}$ ), which are chain homotopy invariants of $C_{*}$. If $Q$ is a factorial ring, each ideal has its greatest common divisor; thus the family of the Fitting ideals yields a family of elements of $Q$, which are called the Fitting invariants of $C_{*}$. When $Q$ is a principal ideal domain, these invariants determine the homology of $C_{*}$ (see Subsection $3.3)$.

If $C_{*}$ is a chain complex over a noncommutative ring $\mathfrak{L}$ and $V$ is a left $Q$-module that is also a right $\mathfrak{L}$-module, we can form the tensor product $V \otimes_{\mathfrak{L}} C_{*}$ and consider the Fitting invariants of the resulting complex. A particular case of this construction leads to the well-known knot polynomials $\Delta_{k}(t)$ of [4, Chapter 8]. We discuss this and similar constructions in Subsections 3.4 and 3.6.

While the definition of the Fitting ideals for chain complexes is apparently new, many similar constructions already exist in the literature. For instance, we mention the invariants of knots deduced from the representation spaces of the fundamental group of the knot (see the paper [33] of Le Ty Kuok Tkhang), and the twisted Alexander-Fox polynomials of Turaev (see [36]).

3.1. Matrices of homomorphisms: terminology. This subsection is purely terminological: we describe the conventions with which we shall be working. Let $R$ be a ring (noncommutative in general). Let $A$ and $B$ be free finitely generated left modules over $R$. Choose a finite basis $\left\{e_{i}\right\}_{1 \leq i \leq k}$ in $A$ and a finite basis $\left\{f_{j}\right\}_{1 \leq j \leq m}$ in $B$. Let $\phi: A \rightarrow B$ be a module homomorphism. Write

$$
\phi\left(e_{i}\right)=\sum_{j} M_{i j} f_{j} \quad \text { with } M_{i j} \in R .
$$

The matrix $\left(M_{i j}\right)$ will be denoted by $M(\phi)$ and called the matrix of the homomorphism $\phi$ with respect to the chosen bases. Thus the coordinates of the images $\phi\left(e_{i}\right)$ of the basis elements of $A$ in $B$ are the rows of the matrix $M(\phi)$ (which has $k$ rows and $m$ columns). Here is the composition formula:

$$
M(\phi \circ \psi)=M(\psi) \cdot M(\phi)
$$

(where $\psi: C \rightarrow A, \phi: A \rightarrow B$ are homomorphisms of left modules and $\cdot$ stands for the usual matrix product). This way of associating a matrix to a module homomorphism will be called row-wise. For a free module $A$ with $k$ free generators, the map

$$
\phi \mapsto M(\phi): \operatorname{Hom}(A, A) \rightarrow \operatorname{Mat}(k \times k, R)
$$

is therefore an antihomomorphism. 
In many recent textbooks on linear algebra, one finds another convention:

$$
\phi\left(e_{i}\right)=\sum_{j} \widetilde{M}_{j i} f_{i}
$$

so that $\widetilde{M}(\phi)=M(\phi)^{T}$, and the coordinates of the images of the basis elements of $A$ in $B$ form the columns of the matrix $\widetilde{M}$. This way of associating the matrix to a module homomorphism will be called column-wise.

When the ring $R$ is commutative, the convention (10) leads to the following composition formula:

therefore the map

$$
\widetilde{M}(\phi \circ \psi)=\widetilde{M}(\phi) \cdot \widetilde{M}(\psi)
$$

is a ring homomorphism.

$$
\phi \mapsto \widetilde{M}(\phi), \quad \operatorname{Hom}(A, A) \rightarrow \operatorname{Mat}(k \times k, R)
$$

\subsection{The Fitting invariants of chain complexes over commutative rings.}

Definition 3.1. A chain complex

$$
C_{*}=\left\{\cdots \longleftarrow C_{k} \stackrel{\partial_{k+1}}{\longleftarrow} C_{k+1} \cdots\right\}
$$

of left modules over a ring $Q$ is called regular if every $C_{i}$ is a finitely generated free $Q$-module, and $C_{i}=0$ for $i<0$.

In this subsection, $Q$ is a commutative factorial ring.

Definition 3.2. Let $C_{*}$ be a regular chain complex of $Q$-modules, and let $k \in \mathbb{N}$. Choose any finite bases in the modules $C_{k}$ and $C_{k+1}$ and let $M\left(\partial_{k+1}\right)$ be the matrix of $\partial_{k+1}$ with respect to these bases.

Let $I_{s}\left(\partial_{k+1}\right)$ denote the ideal in $Q$ generated by all $s \times s$-minors of $M\left(\partial_{k+1}\right)$. (Here we assume that $s$ is an integer with $0<s \leq \min \left(\operatorname{rk} C_{k}, \operatorname{rk} C_{k+1}\right)$. If $s>\min \left(\operatorname{rk} C_{k}, \operatorname{rk} C_{k+1}\right)$, then we put $I_{s}\left(\partial_{k+1}\right)=0$ by definition, and for $s \leq 0$ we put $I_{s}\left(\partial_{k+1}\right)=Q$.)

The following lemma is a well-known consequence of the Binet-Cauchy formula (see, for example, [17, p. 25]).

Lemma 3.3. The ideal $I_{s}\left(\partial_{k+1}\right)$ does not depend on the particular choice of bases in $C_{k}$ and $C_{k+1}$.

It is clear that, in general, the ideal $I_{s}\left(\partial_{k+1}\right)$ is not an invariant of the homotopy type of the chain complex. However, we can reindex the sequence $I_{s}\left(\partial_{k+1}\right)$ and obtain homotopy invariants.

Definition 3.4. Put

$$
J_{m}^{(k)}\left(C_{*}\right)=I_{\mathrm{rk} C_{k}-\mathrm{rk} C_{k-1}-m+1}\left(\partial_{k+1}\right) \cdot^{3}
$$

Proposition 3.5. For any $m$ and $k$, the ideal $J_{m}^{(k)}\left(C_{*}\right)$ is a homotopy invariant of the regular chain complex $C_{*}$.

Proof. Let $F$ be a free finitely generated $Q$-module. Let $T_{*}(i, F)$ denote the chain complex

$$
T_{*}(i, F)=\{0 \longleftarrow \cdots 0 \longleftarrow F \stackrel{\text { id }}{\longleftarrow} F \longleftarrow 0 \longleftarrow \cdots\}
$$

concentrated in degrees $i$ and $i+1$. A chain complex isomorphic to a direct sum of complexes $T_{*}(i, F)$ for some $i \geq 0$ and some $F$ will be called trivial. The following

\footnotetext{
${ }^{3}$ This reindexing may seem arbitrary, but we shall see that it fits with the usual notation for the knot polynomials.
} 
lemma is a version of the Cockcroft-Swan theorem [2], the proof being similar to that of Lemma 1.8 in [24].

Lemma 3.6. Let $C_{*}$ and $D_{*}$ be chain homotopy equivalent complexes. Then there are trivial chain complexes $T_{*}$ and $T_{*}^{\prime}$ such that $C_{*} \oplus T_{*} \approx D_{*} \oplus T_{*}^{\prime}$.

Proof. Let

$$
K_{*}=\bigoplus_{i \in \mathbb{Z}} T\left(i, C_{i}\right)
$$

This is a trivial chain complex. Let $\phi: C_{*} \rightarrow D_{*}$ be a chain homotopy equivalence. Define a chain map $\psi: C_{*} \rightarrow D_{*} \oplus K_{*}$ as follows:

$$
\psi(c)=(\phi(c), c, \partial c) \in D_{i} \oplus C_{i} \oplus C_{i-1} \quad \text { for } c \in C_{i} .
$$

It is clear that $\psi$ is a chain homotopy equivalence that is a split monomorphism. The quotient chain complex $\left(D_{*} \oplus K_{*}\right) / \operatorname{Im} \psi$ is a contractible chain complex of free finitely generated modules. Thus, we obtain an exact sequence

$$
0 \longrightarrow C_{*} \stackrel{\psi}{\longrightarrow} D_{*} \oplus K_{*} \longrightarrow S_{*} \longrightarrow 0,
$$

where $S_{*}$ is a regular acyclic chain complex. Such a sequence splits (see, for example, [3, 13.2]), and we obtain an isomorphism

$$
C_{*} \oplus S_{*} \approx D_{*} \oplus K_{*} .
$$

It is easy to prove that there is a free trivial chain complex $R_{*}$ such that $S_{*} \oplus R_{*}$ is trivial, which completes the proof of the lemma with $T_{*}=S_{*} \oplus R_{*}$ and $T_{*}^{\prime}=K_{*} \oplus R_{*}$.

Now, we return to the proof of our proposition. By the preceding lemma, it suffices to check the following easily proved assertion: the ideal $J_{m}^{(k)}\left(C_{*}\right)$ does not change if we add the chain complex $T_{*}(i, F)$ to $C_{*}$, where $F$ is a finitely generated free $Q$-module, and $i$ equals one of the numbers $k-1, k$ or $k+1$.

Definition 3.7. The ideal $J_{m}^{(k)}\left(C_{*}\right)$ is called the $m$ th Fitting ideal of $C_{*}$. The GCD (= the greatest common divisor) of all nonzero elements in the ideal $J_{m}^{(k)}\left(C_{*}\right)$ will be denoted by $F_{m}^{(k)}\left(C_{*}\right)$ and called the Fitting invariant of $C_{*}$. This element is well defined up to multiplication by invertible elements of $Q$. The sequence

$$
\cdots \subset J_{m}^{(k)}\left(C_{*}\right) \subset J_{m+1}^{(k)}\left(C_{*}\right) \subset \cdots
$$

of the Fitting ideals will be called the Fitting sequence of $C_{*}$ in degree $k$. The subsequence of (13) formed by all nontrivial ideals is called the reduced Fitting sequence of $C_{*}$ in degree $k$.

Recall that an ideal $I \subset Q$ is called nontrivial if $I \neq 0, I \neq Q$.

Observe that the length of the reduced Fitting sequence in degree $k$ is at most $\operatorname{rk} C_{k}$.

Lemma 3.8. Let $S \subset Q^{\bullet}$ be a multiplicative subset of $Q$. Then, up to invertible elements of $S^{-1} Q$, we have

$$
F_{m}^{(k)}\left(C_{*}\right)=F_{m}^{(k)}\left(S^{-1} C_{*}\right)
$$

Proof. It suffices to recall that the GCD of the elements of a factorial ring does not change when the ring is localized.

Remark 3.9. The Fitting invariants $F_{m}^{(k)}$ for $k \leq 2$ can be defined in a slightly more general framework.

Definition 3.10. A chain complex $C_{*}$ of left $Q$-modules will be called 2-regular if $C_{i}=0$ for $i<0$ and $C_{0}, C_{1}$, and $C_{2}$ are finitely generated free $Q$-modules. 
Using the same procedure as above, we can define the Fitting invariants $F_{m}^{(i)}$ with $i \leq 2$ for any 2-regular chain complex over a commutative $\operatorname{ring} Q$.

3.3. The case when $Q$ is principal. Assume that $Q$ is a principal ideal domain. We shall show that in this case the sequence of Fitting invariants determines the homology of the chain complex. We shall give only the statements of the theorems; the proofs are obtained by applying the standard results on the structure of the modules over principal ideal domains.

Let us begin with a theorem which shows how to compute the Betti numbers

$$
b_{k}\left(C_{*}\right)=\operatorname{rk}_{Q} H_{k}\left(C_{*}\right)
$$

and the torsion numbers

$$
q_{k}\left(C_{*}\right)=\text { t.n. } Q H_{k}\left(C_{*}\right)
$$

from the Fitting invariants. (Recall that the torsion number of a module $X$ is the minimal possible number of generators of the torsion submodule of $X$.) Let $C_{*}$ be a regular chain complex of $Q$-modules, and let $\gamma_{k}=\operatorname{rk} C_{k}$. Consider the subsequence of the Fitting sequence starting with $J_{-\gamma_{k-1}+1}^{(k)}=I_{\gamma_{k}}\left(\partial_{k+1}\right)$ :

$$
J_{-\gamma_{k-1}+1}, J_{-\gamma_{k-1}+2}, \ldots
$$

Let $A_{k}$ be the number of zero ideals in this sequence. Let $\varkappa_{k}$ be the cardinality of the reduced Fitting sequence of $C_{*}$ in degree $k$.

Theorem 3.11. We have

$$
\begin{aligned}
& b_{k}\left(C_{*}\right)=A_{k}+A_{k-1}-\gamma_{k-1} ; \\
& q_{k}\left(C_{*}\right)=\varkappa_{k} .
\end{aligned}
$$

The torsion submodule of the homology is also determined by the Fitting invariants. Write the reduced Fitting sequence in degree $k$ as follows:

$$
I_{1}, \ldots, I_{\varkappa_{k}} \text {. }
$$

Let $\theta_{s}$ be the GCD of all the elements of $I_{s}$. Then $\theta_{s+1} \mid \theta_{s}$ for every $s$. Put $\lambda_{s}=\theta_{s} / \theta_{s+1}$.

Theorem 3.12. The elements $\lambda_{s} \in Q$ are noninvertible, for every s we have $\lambda_{s+1} \mid \lambda_{s}$, and

$$
\text { Tors } H_{k}\left(C_{*}\right) \approx \bigoplus_{i=1}^{\varkappa_{k}} Q / \lambda_{i} Q \text {. }
$$

3.4. The twisted Fitting invariants of $\mathbb{Z} G$-complexes. In this subsection, we apply the Fitting invariants of the preceding subsection to construct invariants of chain complexes over noncommutative rings. Modules over group rings are of primary importance for us, and we limit ourselves to this case, although there are obvious generalizations.

Let $G$ be a group, and let $C_{*}$ be a regular chain complex over $\mathfrak{L}=\mathbb{Z} G$. Let $\theta: G \rightarrow$ $\mathrm{GL}_{Q}(W)$ be a right representation, where $W$ is a finitely generated free $Q$-module over a commutative factorial ring $Q$. We form the tensor product

$$
C_{*}(\theta)=W \otimes_{Q} C_{*} ;
$$

the Fitting invariants $F_{m}^{(k)}\left(W \otimes_{Q} C_{*}\right)$ of this complex will be denoted by $\delta_{m}^{(k)}\left(C_{*}, \theta\right)$ and called twisted Fitting invariants of $C_{*}$.

Remark 3.13. As in Remark 3.9, we obtain the Fitting invariants $\delta_{m}^{(k)}\left(C_{*}, \theta\right) \in Q$, where $k \leq 2$ and $C_{*}$ is a 2-regular chain complex. 
In this paper we shall mainly be interested in the case when the ring $Q$ is the group ring of some free Abelian group, or a Novikov completion of such a group ring, or a localization of such a group ring.

Let $H$ be a free Abelian finitely generated group, $R$ a commutative factorial ring. The group ring $R[H]$ will be denoted by $\Lambda$. Let $V$ be a finitely generated free left $R$ module. Let $\rho: G \rightarrow \mathrm{GL}_{R}(V)$ be a right representation of $G$, and let $\pi: G \rightarrow H$ be an epimorphism. Recall that in the Introduction a right representation $\rho_{\pi}: G \rightarrow \operatorname{GL}\left(V^{H}\right)$, where $V^{H}=\Lambda \otimes_{R} V$, was associated with these data.

Definition 3.14. Let $C_{*}$ be a regular chain complex over $\mathbb{Z} G$. The Fitting invariant $\delta_{m}^{(k)}\left(C_{*}, \rho_{\pi}\right) \in \Lambda$ will be called the twisted Fitting invariant of $C_{*}$ with respect to $(\rho, \pi)$.

In the rest of this subsection, we investigate the behavior of the Fitting invariants of $C_{*}$ with respect to certain completions and localizations of the representation $\rho_{\pi}$.

Definition 3.15. Let $\xi: H \rightarrow \mathbb{R}$ be a nonzero homomorphism. An element $x \in \Lambda=$ $R[H]$ is called $\xi$-monic if

$$
x=x_{0} h_{0}+\sum_{i=1}^{s} x_{i} h_{i} \quad \text { with } x_{i} \in R, h_{i} \in H,
$$

where $x_{0} \in R^{\bullet}$ and $\xi\left(h_{i}\right)<\xi\left(h_{0}\right)$ for every $i \neq 0$. The multiplicative subset of all $\xi$-monic elements will be denoted by $S_{\xi}$. The ring $S_{\xi}^{-1} \Lambda$ will also be denoted by $\Lambda_{(\xi)}$.

The next proposition is immediate.

Proposition 3.16. An element $x \in \Lambda$ is $\xi$-monic if and only if it is invertible in $\widehat{\Lambda}_{\xi}$.

Therefore, the ring $\Lambda_{(\xi)}$ can be regarded as a subring of $\widehat{\Lambda}_{\xi}$. Let $\widetilde{\rho}_{\pi, \xi}$ denote the composition

$$
G \longrightarrow \rho_{\pi} \mathrm{GL}_{\Lambda}\left(V^{H}\right) \hookrightarrow \mathrm{GL}_{\Lambda_{(\xi)}}\left(\Lambda_{(\xi)} \otimes_{\Lambda} V^{H}\right) .
$$

This is a right representation of $G$, and we obtain the corresponding twisted Fitting invariants $\delta_{m}^{(k)}\left(C_{*}, \widetilde{\rho}_{\pi, \xi}\right)$. It is clear that

$$
\delta_{m}^{(k)}\left(C_{*}, \rho_{\pi, \xi}\right)=\delta_{m}^{(k)}\left(C_{*}, \widetilde{\rho}_{\pi, \xi}\right) .
$$

Similarly, let $\widehat{\rho}_{\pi, \xi}$ denote the composition

$$
G \longrightarrow \rho_{\pi} \mathrm{GL}_{\Lambda}\left(V^{H}\right) \hookrightarrow \mathrm{GL}_{\widehat{\Lambda}_{\xi}}\left(\widehat{\Lambda}_{\xi} \otimes_{\Lambda} V^{H}\right) .
$$

This is a right representation of $G$, and we obtain the corresponding twisted Fitting invariants $\delta_{m}^{(k)}\left(C_{*}, \widehat{\rho}_{\pi, \xi}\right)$.

In the rest of this subsection, we restrict ourselves to the particular case of $R=\mathbb{Z}$, although some of the results can be proved in a more general setting.

Proposition 3.17. Let $\xi: H \rightarrow \mathbb{R}$ be a monomorphism. Let $R=\mathbb{Z}$. Then

$$
\delta_{m}^{(k)}\left(C_{*}, \rho_{\pi}\right)=\delta_{m}^{(k)}\left(C_{*}, \widehat{\rho}_{\pi, \xi}\right) .
$$

Proof. We shall reduce the proof to formula (17).

Proposition 3.18 (see [21]). If $\xi: H \rightarrow \mathbb{R}$ is monomorphic, then $\Lambda_{(\xi)}$ is a principal ideal domain.

Corollary 3.19. For every two elements $a, b \in \Lambda$, we have

$$
G C D_{\Lambda}(a, b)=G C D_{\Lambda_{(\xi)}}(a, b)=G C D_{\widehat{\Lambda}_{\xi}}(a, b) .
$$

The proposition follows immediately. 
Now we can explain how to compute the twisted Novikov homology of a chain complex in terms of its twisted Fitting invariants. Let $C_{*}$ be a regular chain complex over $\mathfrak{L}=$ $\mathbb{Z} G$. Let $\pi: G \rightarrow H$ be an epimorphism onto a free finitely generated Abelian group, and let $\rho: G \rightarrow \mathrm{GL}(V)$ be a right representation, where $V \approx \mathbb{Z}^{n}$ is a free finitely generated module over $\mathbb{Z}$. Let $\xi: H \rightarrow \mathbb{R}$ be a monomorphism, so that $\widehat{\Lambda}_{\xi}$ is a principal ideal domain. Applying the results of Subsection 3.3, we obtain the following description of the twisted Novikov homology.

Let $\gamma_{k}=n \cdot \operatorname{rk} C_{k}$, where $n=\operatorname{rk}_{\mathbb{Z}} V$, and $\operatorname{rk} C_{k}$ is the number of free generators of $C_{k}$. Consider the segment of the Fitting sequence of $C_{*}\left(\rho_{\pi}\right)=V^{H} \otimes_{\Lambda} C_{*}$ in degree $k$ starting with $J_{-\gamma_{k-1}+1}^{(k)}=I_{\gamma_{k}}\left(\partial_{k+1}\right)$ :

$$
J_{-\gamma_{k-1}+1}, J_{-\gamma_{k-1}+2}, \ldots
$$

Let $A_{k}$ be the number of zero ideals in this segment. Let $I_{1} \subset \ldots \subset I_{B_{k}}$ be the reduced Fitting sequence of $C_{*}\left(\rho_{\pi}\right)$ in degree $k$, and let $\lambda_{s} \in \Lambda$ be the GCD of the ideal $I_{s}$. Then $\lambda_{i} \mid \lambda_{j}$ for $i \geq j$. Let $\varkappa_{k}(\xi)$ be the number of non- $\xi$-monic elements $\lambda_{j}$.

Theorem 3.20. We have

$$
\begin{aligned}
& \widehat{b}_{k}\left(X, \rho_{\pi}, \xi\right)=A_{k}+A_{k-1}-\gamma_{k-1} ; \\
& \widehat{q}_{k}\left(X, \rho_{\pi}, \xi\right)=\varkappa_{k}(\xi) .
\end{aligned}
$$

As for the torsion submodule in degree $k$, let $\theta_{s}=\lambda_{s} / \lambda_{s+1} \in \Lambda$.

Theorem 3.21. The elements $\theta_{s} \in \Lambda_{(\xi)}$ are noninvertible. For every $s$,

$$
\theta_{s+1} \quad \text { divides } \theta_{s} \text { in } \Lambda_{(\xi)}
$$

and

$$
\operatorname{Tors} \widehat{H}_{k}\left(X, \rho_{\pi}, \xi\right) \approx \bigoplus_{i=1}^{\varkappa_{k}(\xi)} \widehat{\Lambda}_{\xi} / \theta_{i} \widehat{\Lambda}_{\xi} .
$$

3.5. The twisted Fitting invariants of $\mathbb{Z} G$-modules. Let $G$ be a group, and let $N$ be a left $\mathbb{Z} G$-module admitting a free finitely generated resolution

$$
0 \longleftarrow R_{0} \longleftarrow R_{1} \longleftarrow R_{2} \longleftarrow \cdots
$$

over $\mathbb{Z} G$ (so that the homology of $R_{*}$ vanishes in all dimensions except zero, and $H_{0}\left(R_{*}\right) \approx$ $N)$. Let $Q$ be a commutative factorial ring, and let $V$ a finitely generated free left $Q$ module. Let $\theta: G \rightarrow \mathrm{GL}(V)$ be a right representation of $G$. Consider the chain complex

$$
V \otimes_{\mathbb{Z} G} R_{*}
$$

of free left $Q$-modules (the module $V$ is endowed with the structure of a right $\mathbb{Z} G$-module via the representation $\theta$ ). Observe that the twisted Fitting invariants

$$
F_{m}^{(k)}\left(V \otimes_{\mathbb{Z} G} R_{*}\right)
$$

depend only on $N$ and $\theta$, but not on the particular choice of the resolution $R_{*}$ (indeed, any two resolutions are chain homotopy equivalent). For us, of principal importance are the Fitting invariants corresponding to the second boundary operator.

Definition 3.22. The element $F_{m}^{(2)}\left(V \otimes_{\mathbb{Z} G} R_{*}\right) \in Q$ will be called the $m$ th Fitting invariant (or det-invariant) of the pair $(G, \theta)$ and denoted by

$$
\delta_{m}(G, N, \theta)=F_{m}^{(2)}\left(V \otimes_{\mathbb{Z} G} R_{*}\right) \in Q .
$$

This element is well defined up to multiplication by an invertible element of the $\operatorname{ring} Q$.

Similarly, we obtain the Fitting invariants $\delta_{m}(G, N, \theta)$ for the case when $N$ is a free left $\mathbb{Z} G$-module admitting a 2-regular resolution (see Remarks 3.9 and 3.13). 
Example 3.23. Let $K$ be an oriented knot; put $G=\pi_{1}\left(S^{3} \backslash K\right)$. Let $N$ be an open tubular neighborhood of $K$. Choose any finite CW decomposition of $S^{3} \backslash N$. The cellular chain complex $\mathcal{C}_{*}\left(\widetilde{S^{3} \backslash N}\right)$ is a free $\mathbb{Z} G$-resolution of the module $H_{0}\left(\widetilde{S^{3} \backslash N}\right) \approx \mathbb{Z}$. The canonical epimorphism $\varepsilon: G \rightarrow \mathbb{Z}$ sending each positively oriented meridian to 1 extends to a ring homomorphism $\theta: \mathbb{Z} G \rightarrow(\mathbb{Z}[\mathbb{Z}])^{\bullet}=\mathrm{GL}(1, \mathbb{Z}[\mathbb{Z}])$. Clearly, the Fitting invariant $\delta_{1}(G, \mathbb{Z}, \theta) \in \mathbb{Z}[\mathbb{Z}]$ equals the Alexander polynomial of the knot. More generally, $\delta_{i}(G, \mathbb{Z}, \theta)$ is the knot polynomial $\Delta_{i}(t)$ (in the terminology of [4, Chapter 8$]$ ).

The previous example has a natural generalization. Let $G$ be a finitely presented group. The Abelian group $\mathbb{Z}$ endowed with the trivial action of $G$ admits a 2-regular free resolution. Let $V$ be a free finitely generated left $R$-module, where $R$ is a commutative factorial ring, and let $\rho: G \rightarrow \mathrm{GL}_{R}(V)$ be a right representation. Let $\pi: G \rightarrow H$ be a homomorphism of $G$ to a free abelian finitely generated group. Then we have the twisted Fitting invariants corresponding to the right representation $\rho_{\pi}: G \rightarrow \mathrm{GL}_{\Lambda}\left(V^{H}\right)$, where $\Lambda=R[H]$.

Definition 3.24. The twisted Fitting invariant $\delta_{m}\left(G, \mathbb{Z}, \rho_{\pi}\right) \in \Lambda$ will also be denoted by $\delta_{m}\left(G, \rho_{\pi}\right)$. The first Fitting invariant $\delta_{1}\left(G, \mathbb{Z}, \rho_{\pi}\right)$ will also be denoted by $A\left(G, \rho_{\pi}\right)$.

It turns out that the Fitting invariants with nonpositive indices vanish.

\section{Lemma 3.25.}

$$
\delta_{m}\left(G, \rho_{\pi}\right)=0 \quad \text { for } m \leq 0 .
$$

Proof. Pick a presentation of a group $G$, let $g_{1}, \ldots, g_{s}$ be the generators, and let $r_{1}, \ldots, r_{l}$ be the relations. Write a 2 -regular free resolution for $\mathbb{Z}$ over $\mathfrak{L}=\mathbb{Z} G$ as follows:

$$
F_{*}=\left\{0 \longleftarrow \mathfrak{L} \stackrel{\partial_{1}}{\longleftarrow} \mathfrak{L}^{s} \stackrel{\partial_{2}}{\longleftarrow} \mathfrak{L}^{l} \longleftarrow \cdots\right\} ;
$$

here the free generators $e_{1}, \ldots, e_{s}$ of the module $F_{1}=\mathfrak{L}^{s}$ correspond to the generators $g_{1}, \ldots, g_{s}$ of $G$ and the homomorphism $\partial_{1}$ is given by $\partial_{1}\left(e_{i}\right)=1-g_{i}$. We can assume that $l \geq s$, and that $t=\pi\left(g_{1}\right)$ is one of the free generators of the Abelian group $H$. We consider the image of our Fitting invariant in the fraction field $\mathcal{R}$ of $R[H]$. This element coincides with the Fitting invariant of the chain complex

$$
\mathcal{F}_{*}=\mathcal{R}^{n} \otimes_{\mathbb{Z} G} F_{*}=\left\{0 \longleftarrow \mathcal{R}^{n} \stackrel{\widetilde{\partial}_{1}}{\longleftarrow} \mathcal{R}^{n s} \stackrel{\widetilde{\partial}_{2}}{\longleftarrow} \mathcal{R}^{n l} \longleftarrow \cdots\right\} .
$$

Observe that $\widetilde{\partial}_{1}$ is an epimorphism. Indeed, the restriction of $\widetilde{\partial}_{1}$ to the first direct summand $\mathcal{R}^{n}$ of $\mathcal{R}^{n s}$ equals $1-t \rho\left(g_{1}\right): \mathcal{R}^{n} \rightarrow \mathcal{R}^{n}$, and the determinant of this map is nonzero, therefore invertible in $\mathcal{R}$. Thus, the rank of the matrix $\widetilde{\partial}_{2}$ is at most $n(s-1)$, and the first Fitting invariant that can be nonzero is

$$
J_{1}^{(2)}\left(\mathcal{F}_{*}\right)=I_{\text {rk } \mathcal{F}_{1}-\mathrm{rk} \mathcal{F}_{0}}\left(\partial_{2}\right) .
$$

The Fitting invariants of modules are useful for computation of the homology with twisted coefficients. Let $X$ be a connected finite CW complex, and put $G=\pi_{1}(X)$. Let $\pi: G \rightarrow H$ be an epimorphism onto a free finitely generated Abelian group, and let $\rho$ : $G \rightarrow \mathrm{GL}(V)$ be a right representation, where $V \approx \mathbb{Z}^{n}$ is a free finitely generated module over $\mathbb{Z}$. Let $\mathcal{C}_{*}(\widetilde{X})$ denote the cellular chain complex of the universal covering of $X$.

Proposition 3.26. $\delta_{i}\left(\mathcal{C}_{*}(\tilde{X}), \rho_{\pi}\right)=\delta_{i}\left(G, \rho_{\pi}\right)$.

Proof. We have

$$
H_{0}(\tilde{X}) \approx \mathbb{Z}, \quad H_{1}(\tilde{X})=0 .
$$

The module $H_{2}(\tilde{X})$ can be nonzero, and therefore the chain complex $\mathcal{C}_{*}(\tilde{X})$ fails in general to be a resolution of the module $\mathbb{Z}$. Choose any subset $S \subset \mathcal{C}_{2}(\widetilde{X})$ generating the 
$\mathbb{Z} G$-submodule $Z_{2}(\widetilde{X})$ of 2 -cycles in the complex $\mathcal{C}_{*}(\tilde{X})$. Put $\mathfrak{L}=\mathbb{Z} G$ and let $\mathfrak{L}^{S}$ be the free $\mathfrak{L}$-module generated by the set $S$. Extend the identity map $S \stackrel{\text { id }}{\longrightarrow} S$ to an $\mathfrak{L}$-module $\operatorname{map} \phi: \mathfrak{L}^{S} \rightarrow \mathcal{C}_{2}(\widetilde{X})$. Put

$$
\mathcal{C}_{3}^{\prime}=\mathcal{C}_{3}(\tilde{X}) \oplus \mathfrak{L}^{S} \quad \text { and } \quad \partial_{3}^{\prime}=\left(\partial_{3}, \phi\right): \mathcal{C}_{3}^{\prime} \rightarrow \mathcal{C}_{2}(\tilde{X}) .
$$

The chain complex $\mathcal{C}_{*}^{\prime}$ is 2-regular, and its second homology vanishes. Applying the same procedure to the third, fourth, etc. homology modules, we obtain an acyclic 2-regular resolution $D_{*}$ of the module $\mathbb{Z}$ such that

$$
\mathcal{C}_{*}(\tilde{X}) \subset D_{*} \quad \text { and } \quad D_{i} / \mathcal{C}_{i}(\tilde{X})=0 \quad \text { for } i \leq 2 .
$$

Thus,

$$
\delta_{i}\left(\mathcal{C}_{*}(\tilde{X}), \rho_{\pi}\right)=\delta_{i}\left(D_{*}, \rho_{\pi}\right) \quad \text { and } \quad \delta_{i}\left(G, \rho_{\pi}\right)=\delta_{i}\left(D_{*}, \rho_{\pi}\right) .
$$

3.6. Fitting invariants of knots and links. Let $L$ be an oriented link. Put $G=$ $\pi_{1}\left(S^{3} \backslash L\right)$. The group $G$ is finitely presented; therefore the module $\mathbb{Z}$ has a 2-regular free resolution over $\mathbb{Z} G$. Thus for any epimorphism $\pi: G \rightarrow H$ and any right representation $\rho: G \rightarrow \mathrm{GL}_{R}(V)$, we obtain a sequence of elements

$$
\delta_{1}\left(G, \rho_{\pi}\right), \delta_{2}\left(G, \rho_{\pi}\right), \ldots \in \Lambda=R[H]
$$

defined up to multiplication by an invertible element of $R$.

Definition 3.27. The elements

$$
\delta_{i}\left(G, \rho_{\pi}\right)=\delta_{i}\left(\pi_{1}\left(S^{3} \backslash L\right), \rho_{\pi}\right)
$$

are called the Fitting invariants of the link $L$.

We shall discuss these invariants and their relationship with Novikov homology in more detail in $\S 5$.

\section{§4. Twisted Alexander polynomials}

We begin with a recollection of M. Wada's definition of the twisted Alexander polynomial (see [37] and also [12]); this occupies the first two subsections. In the rest of the section, we discuss relations between the Fitting invariants and twisted Alexander polynomials. The one-variable case and the multivariable case are slightly different from each other and we consider them separately.

4.1. W-invariant of a matrix. Let $\mathfrak{L}$ be a ring with a unit (noncommutative in general); let $\lambda: \mathfrak{L} \rightarrow \operatorname{Mat}(n \times n, \Lambda)$ be a ring homomorphism, where $\Lambda$ is a commutative factorial ring.

In any matrix $\mathcal{A}$ over $\mathfrak{L}$, we can replace each of its matrix entries $\mathcal{A}_{i j}$ by its image with respect to $\lambda$. The result of this operation will be denoted by $\psi(\mathcal{A}) ;$ the size of $\psi(\mathcal{A})$ is $n$ times the size of $\mathcal{A}$. We have

$$
\psi\left(\mathcal{A}_{1} \mathcal{A}_{2}\right)=\psi\left(\mathcal{A}_{1}\right) \psi\left(\mathcal{A}_{2}\right)
$$

if the number of rows of $\mathcal{A}_{2}$ equals the number of columns of $\mathcal{A}_{1}$.

Now let $\mathcal{B}$ be an $(l \times s)$-matrix with coefficients in $\mathfrak{L}$. Assume that $l \geq s-1$. Let

$$
\alpha=\left(\begin{array}{c}
a_{1} \\
\vdots \\
a_{s}
\end{array}\right)
$$

be a column of elements of $\mathfrak{L}$ such that

$$
\mathcal{B} \cdot \alpha=0 .
$$


We are going to associate an element

$$
W=W(\mathcal{B}, \alpha, \lambda) \in \Lambda
$$

with these data.

For an integer $j$ with $1 \leq j \leq s$, denote by $\mathcal{B}_{j}$ the $l \times(s-1)$-matrix obtained from $\mathcal{B}$ by suppressing the $j$ th column. We have the $(n l \times n(s-1))$-matrix $\psi\left(\mathcal{B}_{j}\right)$. Let $S=i_{1}<i_{2}<\cdots<i_{n(s-1)}$ be a sequence of integers in $[1, n l]$. Let $\psi\left(\mathcal{B}_{j}\right)^{S}$ denote the square matrix formed by all the matrix entries of $\psi\left(\mathcal{B}_{j}\right)$ contained in the rows with indices in $S$. From the condition (22), it is easy to deduce that for every $i$ and $j$ with $1 \leq i, j \leq s$ we have

$$
\operatorname{det}\left(\psi\left(\mathcal{B}_{j}\right)^{S}\right) \cdot \operatorname{det}\left(\psi\left(a_{i}\right)\right)=\operatorname{det}\left(\psi\left(\mathcal{B}_{i}\right)^{S}\right) \cdot \operatorname{det}\left(\psi\left(a_{j}\right)\right) .
$$

Let $Q_{j}(\mathcal{B})$ denote the GCD of the elements $\operatorname{det}\left(\psi\left(\mathcal{B}_{j}\right)^{S}\right)$ over all $S$. This element is defined up to multiplication by an invertible element of $\Lambda$.

Definition 4.1. Assume that there exists $j$ with $\operatorname{det}\left(\psi\left(a_{j}\right)\right) \neq 0$. The element

$$
W(\mathcal{B}, \alpha, \lambda)=\frac{Q_{j}(\mathcal{B})}{\operatorname{det}\left(\psi\left(a_{j}\right)\right)}
$$

of the fraction field of $\Lambda$ will be called the $\mathrm{W}$-invariant of the matrix $\mathcal{B}$.

If $l \leq s-1$, we set $W(\mathcal{B}, \alpha, \lambda)=0$ by definition.

4.2. Twisted Alexander polynomials: definition. Now we apply the construction of the previous subsection to define the twisted Alexander polynomial. Let $G$ be a finitely presented group. Let $\pi: G \rightarrow H$ be an epimorphism of $G$ onto a finitely generated free Abelian group $H$. Let $R$ be a commutative factorial ring, and let $\lambda: G \rightarrow \operatorname{GL}(n, R)$ be a group homomorphism. The tensor product of this homomorphism with $\pi: G \rightarrow R[H]^{\bullet}$ gives rise to a ring homomorphism $\lambda_{\pi}: \mathfrak{L}=\mathbb{Z} G \rightarrow \operatorname{Mat}(n \times n, \Lambda)$, where $\Lambda=R[H]$.

Pick a finite presentation $p=\left(g_{1}, \ldots, g_{s} \mid h_{1}, \ldots, h_{l}\right)$ of the group $G$, with generators $g_{j}$ and relators $h_{i}$. Let $\frac{\partial h_{i}}{\partial g_{j}} \in \mathbb{Z} G$ denote the corresponding Fox derivative.

Definition 4.2. The matrix

$$
\mathcal{A}=\mathcal{A}(G, p)=\left(\frac{\partial h_{i}}{\partial g_{j}}\right) \in \operatorname{Mat}(l \times s, \mathfrak{L})
$$

will be called the Alexander matrix of the presentation $p$.

Let

We have

$$
\alpha=\left(\begin{array}{c}
1-g_{1} \\
\vdots \\
1-g_{s}
\end{array}\right) \in \mathfrak{L}^{s} .
$$

$$
\mathcal{A} \cdot \alpha=0
$$

therefore the constructions of the previous subsection apply and we obtain the $\mathrm{W}$ invariant $W\left(\mathcal{A}, \lambda_{\pi}, \alpha\right)$ in the fraction field of $R[H]$. The ring $R[H]$ being isomorphic to the ring of Laurent polynomials in $k$ variables with coefficients in $R$ (where $k=\mathrm{rk} H$ ), the $\mathrm{W}$-invariant can be viewed as a rational function of $k$ variables with coefficients in the fraction field of $R[H]$. Wada proved in [37] that this element does not depend on the particular choice of the presentation $p$ (up to multiplication by an invertible element of $\Lambda$ ) and, therefore, is determined by $\lambda$ and $\pi$.

Definition 4.3. The element $W\left(\mathcal{A}, \lambda_{\pi}, \alpha\right)$ of the fraction field of $R[H]$ is denoted by $\Delta_{G, \lambda}$ and called the twisted Alexander polynomial of $G$ associated with $\lambda$ and $\pi$. 
4.3. Relationship with the Fitting invariants: the one-variable case. In the case when $H \approx \mathbb{Z}$, the ring $\Lambda=R[H]$ is isomorphic to $R\left[t, t^{-1}\right]$. By definition, the twisted Alexander polynomial is an element of the field of fractions of the ring $R[t]$. Consider a multiplicative subset $\Sigma \subset \Lambda$ consisting of all Laurent polynomials of the form $a_{i} t^{i}+\cdots+a_{j} t^{j}$, where $i, j \in \mathbb{Z}, i \leq j$, and $a_{i}$ and $a_{j}$ are invertible elements of $R$.

Proposition 4.4. $\Delta_{G, \lambda}(t) \in \Sigma^{-1} \Lambda$.

Proof. We can assume that the first generator $g_{1}$ satisfies $\pi\left(g_{1}\right)=t \in R\left[t, t^{-1}\right]$. The first coefficient of the polynomial $\operatorname{det}\left(1-t \lambda\left(g_{1}\right)\right)$ equals 1 , and the last coefficient equals $\pm \operatorname{det}\left(\rho\left(g_{1}\right)\right)$, which is an invertible element of $R$.

Now we can proceed to the comparison of Fitting invariants and twisted Alexander polynomials. Let $\rho: G \rightarrow \mathrm{GL}_{R}(V)$ be a right representation of $G$, where $V$ is a finitely generated free left $R$-module. Choose a basis in $V$; then we obtain a homomorphism

$$
\bar{\rho}: G \rightarrow \mathrm{GL}(n, R) ; \quad \bar{\rho}(g)=M(\rho(g)) .
$$

Proposition 4.5. The Fitting invariant $A\left(G, \rho_{\pi}\right)$ divides the twisted Alexander polynomial $\Delta_{G, \bar{\rho}}$ in the ring $\Sigma^{-1} \Lambda$.

Proof. Let $g_{1}, \ldots, g_{s}$ be generators of $G$, and let $h_{1}, \ldots, h_{l}$ be relators. The free resolution of $\mathbb{Z}$ over $\mathfrak{L}=\mathbb{Z} G$ can be constructed as follows:

$$
F_{*}=\left\{0 \longleftarrow \mathfrak{L} \stackrel{\partial_{1}}{\longleftarrow} \mathfrak{L}^{s} \stackrel{\partial_{2}}{\longleftarrow} \mathfrak{L}^{l} \longleftarrow \cdots\right\},
$$

where

$$
M\left(\partial_{1}\right)=\left(\begin{array}{c}
1-g_{1} \\
\vdots \\
1-g_{s}
\end{array}\right)
$$

and $M\left(\partial_{2}\right)$ equals the Alexander matrix $\mathcal{A}=\mathcal{A}(G, p)$ corresponding to the presentation $p=\left(g_{1}, \ldots, g_{s} \mid h_{1}, \ldots, h_{l}\right)$ (see $\left[1\right.$, Chapter 9]). The polynomial $A\left(G, \rho_{\pi}\right)$ is computed from the chain complex $\mathcal{F}_{*}=\Lambda^{n} \otimes_{\mathbb{Z} G} F_{*}$. The matrix $M\left(\widetilde{\partial}_{2}\right)$ of the second boundary operator in the chain complex $\mathcal{F}_{*}$ is equal to the matrix $\psi(\mathcal{A})$ of Subsection 4.1, where $\psi=\overline{\rho_{\pi}}$. Now the proposition follows easily, since for every $j$ the element $Q_{j}(\mathcal{A})$ in Definition 4.1 is the GCD of a certain family of $((s-1) n \times(s-1) n)$-minors of $M\left(\widetilde{\partial}_{2}\right)$, and $A\left(G, \rho_{\pi}\right)$ is the GCD of all $((s-1) n \times(s-1) n)$-minors.

Proposition 4.6. If the group $G$ has a presentation with $s$ generators and $s-1$ relations (for example, $G$ is the fundamental group of the complement to a link in $S^{3}$ ), then the elements

$$
A\left(G, \rho_{\pi}\right) \quad \text { and } \quad \Delta_{G, \bar{\rho}} \in \Sigma^{-1} \Lambda
$$

are equal up to multiplication by an invertible element of $\Sigma^{-1} \Lambda$.

Proof. As before, we can assume that $\pi\left(g_{1}\right)=t \in R\left[t, t^{-1}\right]$. Suppressing the first $n$ columns of the matrix $\psi(\mathcal{A})$, we obtain an $(n(s-1) \times n(s-1))$-matrix $\mathcal{M}^{\prime}$. Up to invertible elements of $\Sigma^{-1} \Lambda$, we have

$$
\operatorname{det} \mathcal{M}^{\prime}=\Delta_{G, \bar{\rho}}
$$

The boundary operator $\partial_{1}^{\prime}$ in the localized complex

$$
\mathcal{F}_{*}^{\prime}=\Sigma^{-1} \mathcal{F}_{*}=\Sigma^{-1} \Lambda \otimes_{\mathbb{Z} G} F_{*}
$$

is an epimorphism (see $(24)$ for the definition of $F_{*}$ ), and it is easy to deduce that the second boundary operator $\partial_{2}^{\prime}$ in this complex is isomorphic to a homomorphism 
$(0, \phi): \Lambda^{n(s-1)} \rightarrow \Lambda^{n} \oplus \Lambda^{n(s-1)}$ where the matrix of $\phi$ equals $\mathcal{M}^{\prime}$. Thus, up to invertible elements of $\Sigma^{-1} \Lambda$ we have

$$
\operatorname{det} \mathcal{M}^{\prime}=I_{n(s-1)}\left(\partial_{2}\right)=J_{1}^{(2)}\left(\mathcal{F}_{*}^{\prime}\right)=J_{1}^{(2)}\left(\mathcal{F}_{*}\right)=A\left(G, \rho_{\pi}\right),
$$

and the proof is completed.

4.4. Relationship with Fitting invariants: the multivariable case. If $H \approx \mathbb{Z}^{k}$ with $k \geq 2$, the ring $\Lambda=R[H]$ is isomorphic to $R\left[t_{1}, t_{1}^{-1}, \ldots, t_{k}, t_{k}^{-1}\right]$. In this case, the twisted Alexander polynomial is an element of the ring $\Lambda$ itself (cf. [37, p. 253]). Indeed, choose a presentation $p=\left(g_{1}, \ldots, g_{s} \mid h_{1}, \ldots, h_{l}\right)$ of $G$ in such a way that $\pi\left(g_{1}\right)=t_{1}$ and $\pi\left(g_{2}\right)=t_{2}$. Let $\rho: G \rightarrow \mathrm{GL}_{R}(V)$ be a right representation of the group $G$, where $V$ is a free finitely generated $R$-module. Put

$$
P_{1}=\operatorname{det}\left(1-t_{1} \rho\left(g_{1}\right)\right) \text { and } P_{2}=\operatorname{det}\left(1-t_{2} \rho\left(g_{2}\right)\right) .
$$

Then we have

$$
\begin{array}{ll}
P_{1}=1+a_{1} t_{1}+\cdots+a_{n} t_{1}^{n}, & \text { where } a_{n}= \pm \operatorname{det} \rho\left(g_{1}\right) ; \\
P_{2}=1+b_{1} t_{2}+\cdots+b_{n} t_{2}^{n}, & \text { where } b_{n}= \pm \operatorname{det} \rho\left(g_{2}\right) .
\end{array}
$$

Let $\mathcal{A}=\mathcal{A}(G, p)$ denote the Alexander matrix for the presentation $p$. For any sequence $S=i_{1}<i_{2}<\cdots<i_{n(s-1)}$ of integers, put

$$
\alpha_{1}^{S}=\operatorname{det} \psi\left(\mathcal{A}_{1}\right)^{S} \quad \text { and } \quad \alpha_{2}^{S}=\operatorname{det} \psi\left(\mathcal{A}_{2}\right)^{S} .
$$

The property (23) implies that

$$
P_{2} \alpha_{1}^{S}=P_{1} \alpha_{2}^{S} \text { for every } S .
$$

The polynomials $P_{1}$ and $P_{2}$ are relatively prime in $\Lambda$; therefore $P_{1} \mid \alpha_{1}^{S}$ in $\Lambda$, and

$$
\Delta_{G, \bar{\rho}}=\frac{Q_{1}}{P_{1}} \in \Lambda, \quad \text { where } Q_{1}=\operatorname{GCD}_{S} \alpha_{1}^{S},
$$

as we claimed above. Moreover, from (29) it is easy to deduce the following:

$$
\operatorname{GCD}_{S}\left(\alpha_{1}^{S}, \alpha_{2}^{S}\right) \mid \frac{Q_{1}}{P_{1}}
$$

and we obtain the following statement.

Proposition 4.7. The Fitting invariant $A\left(G, \rho_{\pi}\right)$ divides the twisted Alexander polynomial $\Delta_{G, \bar{\rho}}$ in the ring $\Lambda=R[H]$.

Now we proceed to an analog of Proposition 4.6 for the multivariable case. We introduce the corresponding localization.

Definition 4.8. Let $\mu: H \rightarrow \mathbb{R}$ be any nontrivial group homomorphism.

1) We say that an element $x \in \Lambda$ has $\mu$-monic ends if

$$
x=x_{0} h_{0}+\sum_{i=1}^{a-1} x_{i} h_{i}+x_{a} h_{a} \text { with } x_{i} \in R \text { and } h_{i} \in H \text {, where } x_{0}, x_{a} \in
$$
$R^{\bullet}$ and $\mu\left(h_{0}\right)<\mu\left(h_{i}\right)<\mu\left(h_{a}\right), 0<i<a$.

2) The multiplicative subset of all the elements of $\Lambda$ with $\mu$-monic ends will be denoted by $\Sigma_{\mu} \subset \Lambda$.

Proposition 4.9. Assume that the group $G$ has a presentation with s generators and $s-1$ relators. Then the images of the elements $A\left(G, \rho_{\pi}\right)$ and $\Delta_{G, \bar{\rho}}$ in the ring $\Sigma_{\mu}^{-1} \Lambda$ are equal up to invertible elements of this ring.

Proof. Similar to the proof of Proposition 4.6. 
It is natural to ask whether the Fitting invariant and twisted Alexander polynomial are equal up to invertible elements of $\Lambda$, at least in the case of knot groups. I do not know if this is true.

\section{§5. Three-Dimensional MANIFOLDS}

In this section, we study the particular case of $C^{\infty}$ compact three-dimensional manifolds $M$ of zero Euler characteristic. We prove Theorem 5.5, which gives a criterion for the vanishing of the twisted Novikov homology of $M$ in terms of the twisted Alexander polynomial of $\pi_{1}(M)$ or, equivalently, in terms of the first Fitting invariant of $\pi_{1}(M)$. The second main result of this section is Theorem 5.7. For a given right representation $\rho$ of $\pi_{1}(M)$ and for a given epimorphism $\pi_{1}(M) \rightarrow H$, this theorem describes the structure of all classes $\xi \in H^{1}(M, \mathbb{R})$ such that the $\rho$-twisted Novikov homology $\widehat{H}_{*}\left(M, \rho_{\pi}, \xi\right)$ vanishes. It turns out that this set is an open conical polyhedral subset of $H^{1}(M, \mathbb{R})$. In Subsection 5.3, we discuss the relationship between the twisted Novikov homology and the Thurston norm; we also suggest a natural question about this relationship.

\subsection{The twisted Novikov homology and the twisted Alexander polynomial} for 3-manifolds. Let $M$ be a compact $C^{\infty}$ three-dimensional manifold with $\chi(M)=0$. Write $G=\pi_{1}(M)$ and $\mathfrak{L}=\mathbb{Z} G$. We begin with two lemmas, which describe the homotopy type of the chain complex of the universal covering of $M$.

Lemma 5.1. Let $M$ be a closed connected 3-manifold. Then the cellular chain complex of its universal covering is chain homotopy equivalent to the chain

$$
C_{*}=\left\{0 \longleftarrow \mathfrak{L} \stackrel{\partial_{1}}{\longleftarrow} \mathfrak{L}^{l} \stackrel{\partial_{2}}{\longleftarrow} \mathfrak{L}^{l} \stackrel{\partial_{3}}{\longleftarrow} \mathfrak{L} \longleftarrow 0\right\},
$$

where:

1) The matrix of $\partial_{1}$ equals

$$
\left(\begin{array}{c}
1-g_{1} \\
1-g_{2} \\
\vdots \\
1-g_{l}
\end{array}\right)
$$

and the elements $g_{1}, \ldots, g_{l} \in G$ generate the group $G$.

2) The matrix of $\partial_{3}$ equals

$$
\left(1-\varepsilon_{1} h_{1}, \quad 1-\varepsilon_{2} h_{2}, \quad \ldots, \quad 1-\varepsilon_{l} h_{l}\right),
$$

where $h_{i} \in G$ and $\varepsilon_{i}=1$ if the loop $h_{i}$ preserves the orientation, and $\varepsilon_{i}=-1$ if $h_{i}$ reverses the orientation. The elements $h_{1}, \ldots, h_{l} \in G$ generate the group $G$.

3) The matrix of $\partial_{2}$ is the Alexander matrix associated with some presentation of the group $G$.

Proof. The lemma follows immediately from the existence of the Heegaard decomposition for closed three-dimensional manifolds.

Lemma 5.2. Let $M$ be a connected compact 3-manifold with nonempty boundary and $\chi(M)=0$. Then the cellular chain complex of the universal covering $\widetilde{M}$ is chain homotopy equivalent to the following one:

$$
C_{*}=\left\{0 \longleftarrow \mathfrak{L} \stackrel{\partial_{1}}{\longleftarrow} \mathfrak{L}^{l} \stackrel{\partial_{2}}{\longleftarrow} \mathfrak{L}^{l-1} \longleftarrow 0\right\},
$$


where the matrix of $\partial_{1}$ equals

$$
\left(\begin{array}{c}
1-g_{1} \\
1-g_{2} \\
\vdots \\
1-g_{l}
\end{array}\right)
$$

and the elements $g_{1}, \ldots, g_{l} \in G$ generate the group $G$. The matrix of $\partial_{2}$ is the Alexander matrix associated with some presentation of the group $G$.

Proof. Morse theory guarantees the existence of a Morse function $f: M \rightarrow \mathbb{R}$ such that

$$
f \mid \partial M=\text { const }=\max _{x \in M} f(x)
$$

and the number $m_{i}(f)$ of critical points of index $i$ satisfies the following:

$$
m_{0}(f)=1, \quad m_{1}(f)=m_{2}(f), \quad m_{3}(f)=0 .
$$

The cellular decomposition corresponding to $f$ satisfies the requirements of the lemma.

We shall use these lemmas to study the twisted Alexander polynomial and the Fitting invariants of $M$. Let $V$ be a free finitely generated $R$-module (where $R$ is a commutative factorial ring), and let $\rho: G \rightarrow \mathrm{GL}_{R}(V)$ be a right representation. Let $\pi: G \rightarrow H$ be an epimorphism of $G$ onto a free Abelian finitely generated group $H$. Let $\xi: H \rightarrow \mathbb{R}$ be any nontrivial homomorphism. Denote $R[H]$ by $\Lambda$, and let $\Sigma_{\xi}$ be the multiplicative subset of Laurent polynomials with $\xi$-monic ends (see Definition 4.8). Let

$$
\widetilde{C}_{*}=\Sigma_{\xi}^{-1}\left(V^{H} \otimes_{\mathfrak{L}} C_{*}\right),
$$

where $V^{H}$ is endowed with the structure of a right $\mathfrak{L}$-module determined by the representation $\rho_{\pi}: G \rightarrow \operatorname{GL}\left(V^{H}\right)$. Put $\Lambda_{[\xi]}=\Sigma_{\xi}^{-1} \Lambda$, and denote rk $V$ by $n$. We have a natural isomorphism

$$
\widetilde{C}_{1} \approx \Lambda_{[\xi]}^{n(l-1)} \oplus \Lambda_{[\xi]}^{n}
$$

(where the first summand of the direct sum corresponds to the elements $e_{1}, \ldots, e_{l-1}$ of the $\mathfrak{L}$-basis of $C_{1}$, and the second summand corresponds to $e_{l}$ ). The projection of $\widetilde{C}_{1}$ onto the direct summand $\Lambda_{[\xi]}^{n(l-1)}$ will be denoted by $p_{1}$. Similarly, the module $\widetilde{C}_{2}$ is naturally isomorphic to $\Lambda_{[\xi]}^{n(l-1)}$ in the case where $\partial M \neq \varnothing$ and to the direct $\operatorname{sum} \Lambda_{[\xi]}^{n(l-1)} \oplus \Lambda_{[\xi]}^{n}$ in the case of closed manifolds. Let

$$
\mathcal{D}=p_{1} \circ\left(\partial_{2} \mid \Lambda_{[\xi]}^{n(l-1)}\right) .
$$

Proposition 5.3. Assume that

$$
\xi\left(g_{l}\right)<0
$$

and in the case where $\partial_{1}(M)=\varnothing$ assume that, moreover,

$$
\xi\left(h_{l}\right)<0
$$

Then the chain complex $\widetilde{C}_{*}$ is chain homotopy equivalent to a free $\Lambda_{[\xi]}$-chain complex

$$
0 \longleftarrow \Lambda_{[\xi]}^{n(l-1)} \stackrel{\mathcal{D}}{\longleftarrow} \Lambda_{[\xi]}^{n(l-1)} \longleftarrow 0
$$

concentrated in dimensions 1 and 2 . 
Proof. We treat the case of closed manifolds, the case $\partial M \neq 0$ being similar. Observe that the homomorphisms

$$
1-\rho_{\pi}\left(g_{l}\right), \quad 1-\rho_{\pi}\left(h_{l}\right): \Lambda_{[\xi]}^{n} \rightarrow \Lambda_{[\xi]}^{n}
$$

are invertible. Therefore, using a standard basis change in the chain complex $\widetilde{C}_{*}$, we can split off from $\widetilde{C}_{*}$ two trivial chain complexes

$$
A_{*} \approx T_{*}\left(1, \Lambda_{[\xi]}^{n}\right) \quad \text { and } \quad B_{*} \approx T_{*}\left(2, \Lambda_{[\xi]}^{n}\right),
$$

in such a way that the resulting chain complex is isomorphic to (35).

Now we can establish the relationship between the twisted Alexander polynomial and the Fitting invariant of a three-manifold.

Proposition 5.4. Let $G$ be the fundamental group of a compact three-dimensional $C^{\infty}$ manifold $M$ with $\chi(M)=0$. Let $\rho: G \rightarrow \mathrm{GL}_{R}(V)$ be a right representation, where $V$ is a free finitely generated $R$-module over a factorial commutative ring $R$. Let $\pi: G \rightarrow H$ be an epimorphism, where $H$ is a free Abelian finitely generated group. Let $\xi: H \rightarrow \mathbb{R}$ be any nontrivial homomorphism.

Then the Fitting invariant $A\left(G, \rho_{\pi}\right)$ and the twisted Alexander polynomial $\Delta_{G, \bar{\rho}}$ are equal up to multiplication by an invertible element of $\Lambda_{[\xi]}$.

Proof. We shall treat the case of closed manifolds, while the case where $\partial M \neq \varnothing$ is even simpler, and we omit it.

The Fitting invariant of a chain complex depends only on its homotopy type and does not change when we localize the base ring. Therefore, we can use the chain complex (35) for computation of the image of $A\left(G, \rho_{\pi}\right)$ in the ring $\Lambda_{[\xi]}$ (we assume that the conditions (33) and (34) hold true, which is easy to arrange by a permutation of the elements of the basis). Thus, the image of $A\left(G, \rho_{\pi}\right)$ in the $\operatorname{ring} \Lambda_{[\xi]}$ equals $\operatorname{det} \mathcal{D}$ (up to invertible elements of this ring).

To compute the twisted Alexander polynomial, we use the matrix of the boundary operator $\partial_{2}$ in $(30)$. The $(n l \times n l)$-matrix $\mathcal{D}^{\prime}=\psi\left(\partial_{2}\right)$ satisfies $\mathcal{H} \circ \mathcal{D}^{\prime}=0$, where $\mathcal{H}=\psi\left(\left(1-h_{1}, \ldots, 1-h_{l}\right)\right)$.

Let $\mathcal{D}^{\prime \prime}$ denote the $(n l \times n(l-1))$-matrix obtained by suppressing the last $n$ columns of the matrix of $\partial_{2}$. Using the invertibility over $\Lambda_{[\xi]}$ of the matrix $1-\rho_{\pi}\left(h_{l}\right)$ and the condition $\mathcal{D}^{\prime \prime} \circ \mathcal{H}=0$, we deduce that the last $n$ rows of the matrix $\mathcal{D}^{\prime \prime}$ are linear combinations of the first $n(l-1)$ rows. This implies that the GCD of the $(n(l-1) \times$ $n(l-1)$ )-minors of the matrix $\mathcal{D}^{\prime \prime}$ equals the determinant of $\mathcal{D}$. Therefore, we obtain the following identity:

and the proof is finished.

$$
\Delta_{G, \bar{\rho}}=\frac{\operatorname{det} \mathcal{D}}{\operatorname{det}\left(1-\rho_{\pi}\left(g_{l}\right)\right)},
$$

Theorem 5.5. Let $M$ be a connected compact $C^{\infty}$ manifold of dimension 3 with $\chi(M)=$ 0 . Let $G=\pi_{1}(M)$, and let $\rho: G \rightarrow \mathrm{GL}_{R}(V)$ be a right representation of the group $G$, where $V$ is a finitely generated free module over a commutative factorial ring $R$. Let $\pi: G \rightarrow H$ be an epimorphism onto a free Abelian finitely generated group, and let $\xi: H \rightarrow \mathbb{R}$ be a nonzero homomorphism. Then the following four conditions are equivalent.

1) The twisted Novikov homology $\widehat{H}_{i}\left(M, \rho_{\pi}, \xi\right)$ vanishes for all $i$.

2) The first twisted Novikov homology module $\widehat{H}_{1}\left(M, \rho_{\pi}, \xi\right)$ vanishes.

3) The Fitting invariant $A\left(G, \rho_{\pi}\right)$ is $\xi$-monic.

4) The twisted Alexander polynomial $\Delta_{G, \bar{\rho}}$ is $\xi$-monic. 
Proof. Observe that the last two conditions are equivalent in view of Proposition 5.4. Therefore, it suffices to prove that the first three conditions are equivalent. Proposition 5.3 implies that the twisted Novikov homology $\widehat{H}_{*}\left(M, \rho_{\pi}, \xi\right)$ is isomorphic to the homology of the chain complex

$$
0 \longleftarrow \widehat{\Lambda}_{\xi}^{n(l-1)} \stackrel{\operatorname{id} \otimes \mathcal{D}}{\longleftarrow} \widehat{\Lambda}_{\xi}^{n(l-1)} \longleftarrow 0,
$$

concentrated in dimensions 1 and 2 , and $\operatorname{det} \mathcal{D}$ equals the Fitting invariant of $M$ with respect to $\rho$ and $\xi$. The homology of the chain complex (36) vanishes for every $i$ if and only if it vanishes for $i=1$, and both of these conditions are equivalent to the invertibility of $\operatorname{det} \mathcal{D}$ in $\widehat{\Lambda}_{\xi}$. This latter condition holds true if and only if this determinant is invertible in $\Lambda_{(\xi)}$. In the course of the proof of Proposition 5.4, we saw that the elements $\operatorname{det} \mathcal{D}$ and $A\left(G, \rho_{\pi}\right)$ are equal up to multiplication by invertible elements of $\Lambda_{(\xi)}$. Therefore, the twisted Novikov homology vanishes if and only if the element $A\left(G, \rho_{\pi}\right)$ is $\xi$-monic.

The previous theorem allows us to describe the structure of the set of all $\xi$ such that the Novikov homology $\widehat{H}_{*}\left(M, \rho_{\pi}, \xi\right)$ vanishes.

Definition 5.6. Let $G=\pi_{1}(M)$, and let $\rho: G \rightarrow \mathrm{GL}_{R}(V)$ be a right representation of the group $G$. Let $\pi: G \rightarrow H$ be an epimorphism onto a free Abelian finitely generated group. A nonzero cohomology class $\xi \in H^{1}(M, \mathbb{R})$ will be called $(\rho, \pi)$-acyclic if the twisted Novikov homology $\widehat{H}_{1}\left(M, \rho_{\pi}, \xi\right)$ vanishes. When the homomorphism $\pi$ is clear from the context, we shall say that $\xi$ is $\rho$-acyclic.

Theorem 5.7. For a given right representation $\rho: G \rightarrow \mathrm{GL}_{R}(V)$ and a given epimorphism $\pi: G \rightarrow H$, the set of all $(\rho, \pi)$-acyclic classes $\xi \in H^{1}(M, \mathbb{R})$ is an open polyhedral conical subset of $H^{1}(M, \mathbb{R})$. If $R$ is a field, then the set of all $(\rho, \pi)$-acyclic classes is either empty, or equals $H^{1}(M, \mathbb{R}) \backslash\{0\}$, or is the complement in $H^{1}(M, \mathbb{R})$ to a finite union of integral hyperplanes.

Proof. The Fitting invariant $A\left(G, \rho_{\pi}\right)$ is an element of $R[H]$. The group $H$ is isomorphic to the integral lattice $\mathbb{Z}^{k} \subset \mathbb{R}^{k} \approx H \otimes \mathbb{R}$, and $A\left(G, \rho_{\pi}\right)$ is then identified with a Laurent polynomial $\mathcal{A}$ in the variables $t_{1}, \ldots, t_{k}$ with coefficients in $R$. If $\mathcal{A}=0$, then no class $\xi$ is $\rho$-acyclic. If $\mathcal{A}$ is a monomial, $\mathcal{A}=\alpha \cdot h$, where $\alpha \in R$, and $h \in H$, then either

(1) $\alpha$ is invertible, and in this case all classes $\xi$ are $\rho$-acyclic, or

(2) $\alpha$ is noninvertible, and in this case there are no $\rho$-acyclic classes.

Now, we consider the nondegenerate case when the Newton polytope $\mathcal{P}$ of the polynomial $\mathcal{A}$ contains more than one point. For a homomorphism $\xi: H \rightarrow \mathbb{R}$, the polynomial $\mathcal{A}$ is $\xi$-monic if and only if the polytope $\mathcal{P}$ has a vertex $v$ such that:

1) the coefficient $a_{v}$ of $\mathcal{A}$ corresponding to this vertex is an invertible element of $R$,

2) for every other vertex $v^{\prime}$ of $\mathcal{P}$ we have $\xi(v)>\xi\left(v^{\prime}\right)$.

For a given vertex $v$, the set $\Gamma_{v}$ of all $\xi$ satisfying conditions 1) and 2) above is an open polyhedral cone. Indeed, for a pair of vertices $v, v^{\prime}$ of $\mathcal{P}$, put

$$
\Gamma_{v, v^{\prime}}=\left\{\xi \in H^{1}(M, \mathbb{R}) \mid \xi(v)=\xi\left(v^{\prime}\right)\right\}
$$

and

$$
\Gamma_{v, v^{\prime}}^{+}=\left\{\xi \in H^{1}(M, \mathbb{R}) \mid \xi(v)>\xi\left(v^{\prime}\right)\right\},
$$

so that $\Gamma_{v, v^{\prime}}^{+}$is one of two open half-spaces corresponding to $\Gamma_{v, v^{\prime}}$. Then

$$
\Gamma_{v}=\bigcap_{v^{\prime}} \Gamma_{v, v^{\prime}}^{+} .
$$

The sets $\Gamma_{v}$ are open polyhedral cones, which are disjoint for different $v$. 
Now let $R$ be a field. The cases when $\mathcal{A}=0$ or $\mathcal{A}$ is invertible are treated as above. When the Newton polytope $\mathcal{P}$ of $\mathcal{A}$ contains more than one vertex, the set of all $(\rho, \pi)$ acyclic classes is the complement in $H^{1}(M, \mathbb{R})$ to the union of all the hyperplanes $\Gamma_{v, v^{\prime}}$.

5.2. Detecting fibered links. In this subsection we give a necessary condition for a link in $S^{3}$ to be fibered. First, we recall the definition of a fibered link and related notions.

Definition 5.8. 1. Let $V$ be a compact topological $(n-1)$-manifold with $\partial V \neq \varnothing$, and let $h: V \rightarrow V$ be a homeomorphism that restricts to the identity on $\partial V$. Forming a mapping torus $V_{h}$ and identifying $(x, t) \sim\left(x, t^{\prime}\right)$ for each $x \in \partial V$ and $t, t^{\prime} \in S^{1}$, we obtain a closed topological manifold. This manifold is denoted by $B(V, h)$.

A closed manifold $M$ is called an open book decomposition if it is homeomorphic to $B(V, h)$ for some $V$ and $h$. The images in $M$ of fibers $V \times\{t\}\left(t \in S^{1}\right)$ are called pages of the open book, and the image of $\partial V \times\{t\}$ in $M$ is called the binding.

2. A $C^{\infty}$-embedding of the disjoint union of several copies of an oriented circle into $S^{3}$ is called an oriented link.

3. An oriented link $L$ is said to be fibered if there is an orientation-preserving homeomorphism

$$
\phi: S^{3} \rightarrow B\left(F^{2}, h\right),
$$

where $F^{2}$ is a compact oriented surface and the restriction of $\phi$ to $L$ is an orientation-preserving homeomorphism onto the binding $\partial F$ of the open book.

For an oriented link $L$, let $G=\pi_{1}\left(S^{3} \backslash L\right)$. Let $\eta: G \rightarrow \mathbb{Z}$ be a homomorphism that sends every positive meridian of $L$ to $1 \in \mathbb{Z}$. Form the corresponding completion $\widehat{\mathfrak{L}}_{\eta}$ of the group ring $\mathfrak{L}=\mathbb{Z} G$, and let $\mathcal{C}_{*}\left(\widetilde{S^{3} \backslash L}\right)$ be the cellular chain complex of the universal covering of the link complement.

If the link $L$ is fibered, then the complement $S^{3} \backslash L$ admits a fibration over a circle. Although the manifold $S^{3} \backslash L$ is not compact, it turns out that the corresponding analog of Theorem 2.5 holds true; this is the subject of the following proposition.

Proposition 5.9. If the link $L$ is fibered, then

$$
H_{*}\left(\widehat{\mathfrak{L}}_{\eta} \otimes_{\mathfrak{L}} \mathcal{C}_{*}\left(\widetilde{S^{3} \backslash L}\right)\right)=0 .
$$

Proof. It is clear that $S^{3} \backslash L$ is homotopy equivalent to the mapping torus $F_{h}^{2}$, where $h$ is a homeomorphism. The space $F_{h}^{2}$ is homotopy equivalent to the mapping torus $F_{g}^{2}$, where $g: F^{2} \rightarrow F^{2}$ is a cellular map (see [28, Proposition 6.1]). Thus, our proposition is a consequence of the following theorem.

Theorem 5.10. Let $X$ be a finite connected $C W$-complex. Let $g: X \rightarrow X$ be a cellular map, and let $X_{g}$ be the mapping torus. Let $G=\pi_{1}\left(X_{g}\right)$, and let $\eta: G \rightarrow \mathbb{Z}$ be the homomorphism induced by the projection $f: X_{g} \rightarrow S^{1}$. Put $\mathfrak{L}=\mathbb{Z} G$ and let $\widehat{\mathfrak{L}}_{\eta}$ be the corresponding Novikov completion. Then

$$
H_{*}\left(\widehat{\mathfrak{L}}_{\eta} \otimes_{\mathfrak{L}} \mathcal{C}_{*}\left(\widetilde{X_{g}}\right)\right)=0 .
$$

Proof. Let $H=\operatorname{ker} \eta, R=\mathbb{Z} H$, and

$$
\begin{aligned}
& \left.\left.P=\left\{\lambda \in \mathfrak{L} \mid \sup \lambda \in \eta^{-1}(]-\infty, 0\right]\right)\right\}, \\
& \left.\left.\widehat{P}=\left\{\lambda \in \widehat{\mathfrak{L}}_{\eta} \mid \sup \lambda \in \eta^{-1}(]-\infty, 0\right]\right)\right\} .
\end{aligned}
$$


Pick any $t \in G$ such that $\eta(t)=-1$. Then the $\operatorname{ring} P$ is isomorphic to the twisted polynomial ring $R_{\theta}[t]$, and the ring $\widehat{P}$ is isomorphic to the twisted power series ring $R_{\theta}[[t]]$ (where $\theta$ is the isomorphism of the ring $R$ defined by $x \mapsto t x t^{-1}$ ). Denote by $Y \rightarrow X_{g}$ the infinite cyclic covering induced by the natural projection $f: X_{g} \rightarrow S^{1}$ from the universal covering $\mathbb{R} \rightarrow S^{1}$. The function $f$ lifts to a continuous function $F: Y \rightarrow \mathbb{R}$; for each $k \in \mathbb{Z}$ the space $F^{-1}([k, k+1])$ is homeomorphic to the mapping cylinder $Z_{g}$ of the map $g: X \rightarrow X$. Let $\left.\left.Y_{k}=F^{-1}(]-\infty, k\right]\right)$. For every $m<k$, the inclusion $Y_{m} \subset Y_{k}$ is a homotopy equivalence. Let $\widetilde{Y}$ be the universal covering for $Y$, which is also a universal covering for $X_{g}$. Let $\widetilde{Y}_{k}$ be the inverse image of $Y_{k}$ in $\widetilde{Y}$.

The cellular chain complex $\mathcal{C}_{*}\left(\widetilde{Y}_{k}\right)$ is a free finitely generated $P$-module. For every $k$, we have

$$
\widehat{\mathfrak{L}}_{\eta} \otimes_{\mathfrak{L}} \mathcal{C}_{*}(\widetilde{Y})=\widehat{\mathfrak{L}}_{\eta} \otimes_{\widehat{P}}\left(\widehat{P} \otimes_{P} \mathcal{C}_{*}\left(\widetilde{Y}_{k}\right)\right)
$$

thus it remains to show that the chain complex $\widehat{P} \otimes_{P} \mathcal{C}_{*}\left(\widetilde{Y}_{0}\right)$ is acyclic. Observe that, since the modules $\mathcal{C}_{*}\left(\widetilde{Y}_{k}\right)$ are free finitely generated $P$-modules, we have

$$
\widehat{P} \otimes_{P} \mathcal{C}_{*}\left(\widetilde{X}_{0}\right)=\lim _{\longleftarrow} \mathcal{C}_{*}\left(\widetilde{X}_{0}, \widetilde{X}_{-k}\right)
$$

(where $k \in \mathbb{N}$ ). Since the inclusion $X_{-k} \hookrightarrow X_{0}$ is a homotopy equivalence, all chain complexes $\mathcal{C}_{*}\left(\widetilde{X}_{0}, \widetilde{X}_{-k}\right)$ are acyclic.

Applying Theorem A.19 of [16], Appendix, we obtain:

$$
H_{*}\left(\lim \mathcal{C}_{*}\left(\tilde{Y}_{0}, \tilde{Y}_{-k}\right)\right) \approx \lim _{\longleftarrow} H_{*}\left(\mathcal{C}_{*}\left(\tilde{Y}_{0}, \tilde{Y}_{-k}\right)\right)=0,
$$

which completes the proof.

Now we apply this result to the twisted Novikov homology of the complement to a fibered link. Let $R$ be a commutative ring. Denote by $\xi$ the inclusion $\mathbb{Z} \hookrightarrow \mathbb{R}$, and put $\Lambda=R[\mathbb{Z}]$. Then the ring $\widehat{\Lambda}_{\xi}$ is identified with the ring $R((t))$, and the ring $\Lambda_{(\xi)}$ is identified with the ring

$$
R\langle t\rangle=\left\{\frac{P(t)}{t^{n} \cdot(1+t Q(t))} \mid n \in \mathbb{Z} \text { and } P, Q \in R[t]\right\} \subset R((t)) .
$$

The $\xi$-monic elements of $\Lambda$ are identified with polynomials of the form $\mu(1+t Q(t))$, where $Q(t) \in R[t]$, and $\mu \in R$ is invertible. Invertible elements of $\Lambda_{(\xi)}=R\langle t\rangle$ are also called monic. Let $\rho: G \rightarrow \mathrm{GL}_{R}(V)$ be any right representation.

Proposition 5.11. If $L$ is fibered, then the twisted Novikov homology $\widehat{H}_{*}\left(S^{3} \backslash L, \rho_{\pi}, \xi\right)$ equals zero.

Proof. The proposition is deduced from Theorem 5.10 in the same way as Theorem 2.8 is deduced from Theorem 2.5.

Observe that the space $S^{3} \backslash L$ is homotopy equivalent to a compact 3-manifold with boundary. Indeed, let $N$ be an open tubular neighborhood of $L$ in $S^{3}$. Then $S^{3} \backslash L$ has the same homotopy type as $S^{3} \backslash N$. Theorem 5.5 implies the following result.

Corollary 5.12. Let $G$ denote the group $\pi_{1}\left(S^{3} \backslash L\right)$. If the link $L$ is fibered, then:

(1) the Fitting invariant $A\left(G, \rho_{\pi}\right) \in R\left[t, t^{-1}\right]$ is monic;

(2) the twisted Alexander polynomial $\Delta_{G, \bar{\rho}} \in R\langle t\rangle$ is monic. 
This result is related to the theorem due to Goda, Kitano, and Morifuji [9]. Their theorem says that if a knot $K$ is fibered and $\rho: \pi_{1}\left(S^{3} \backslash K\right) \rightarrow S L(n, F)$ is a representation (where $F$ is a field), then the leading coefficient of the twisted Alexander polynomial associated with $\rho$ equals 1 .

Our theorem is valid in a more general setting: it allows representations in $\operatorname{GL}(n, R)$, where $R$ is a factorial ring, and not only in $S L(n, F)$. On the other hand, for the representations in $S L(n, F)$, the theorem of [9] gives much more information because it guarantees that the leading coefficient of the twisted Alexander polynomial equals 1 , while Corollary 5.12 asserts only that this coefficient is nonzero, that is, the twisted Alexander polynomial does not vanish.

5.3. Thurston cones and $\rho$-acyclicity cones. Let $M$ be a closed three-dimensional $C^{\infty}$ manifold. Put $G=\pi_{1}(M), \mathfrak{L}=\mathbb{Z} G$. Let $\mathcal{V}(M) \subset H^{1}(M, \mathbb{R})$ be the subset of all cohomology classes representable by closed 1-forms without zeros. Let

$$
\mathcal{V}_{h}(M)=\left\{\xi \in H^{1}(M, \mathbb{R}) \mid H_{*}\left(\widehat{\mathfrak{L}}_{\xi} \otimes_{\mathfrak{L}} \mathcal{C}_{*}(\widetilde{M})\right)=0\right\} .
$$

For a given right representation $\rho: \pi_{1}(M) \rightarrow \operatorname{GL}\left(\mathbb{Z}^{n}\right)$, let $\mathcal{V}_{\text {alg }}(M, \rho)$ be the subset of all $(\rho, \pi)$-acyclic cohomology classes $\xi$, where $\pi$ is the projection $\pi_{1}(M) \rightarrow H_{1}(M) /$ Tors. From the results of the previous section, it follows that

$$
\mathcal{V}(M) \subset \mathcal{V}_{h}(M) \subset \mathcal{V}_{\text {alg }}(M)=\bigcap_{\rho \in \mathcal{R}} \mathcal{V}_{\text {alg }}(M, \rho),
$$

where $\mathcal{R}$ is the set of all right representations $\pi_{1}(M) \rightarrow \mathrm{GL}\left(\mathbb{Z}^{n}\right)$. The Thurston theorem [34] implies that the set $\mathcal{V}(M)$ is an open conical polyhedral subset of $H^{1}(M, \mathbb{R})$. The set $\mathcal{V}_{\text {alg }}(M, \rho)$ is also an open conical polyhedral subset of $H^{1}(M, \mathbb{R})$, as it follows from the results of the previous section.

Question. For which manifolds $M$ are the inclusions (37) equalities?

For every $\rho$, the set $\mathcal{V}_{\text {alg }}(M, \rho)$ is effectively computable from the twisted Alexander polynomial or from the first Fitting invariant. Thus, the investigation of the inclusions (37) will give much information on the structure of the set $\mathcal{V}(M)$. We think that computer experiments can help here, and can clarify the problem (see paper [11] for an example of application of Kodama's program KNOT to a similar question). In the rest of this section, we shall show that the properties of the inclusion (37) are quite sensitive to the class of the representations that we consider. Let $\mathcal{R}_{\mathcal{F}}$ be the set of all representations $G \rightarrow \operatorname{GL}\left(\mathbf{F}^{n}\right)$, where $\mathbf{F}$ is a finite field. We are going to show that there are closed manifolds $M$ with

$$
\mathcal{V}(M) \neq \bigcap_{\rho \in \mathcal{R}_{\mathcal{F}}} \mathcal{V}_{\text {alg }}(M, \rho)
$$

Let $M$ be any 3-manifold with the following property $(L)$ :

L1) there is an open subset $U \subset H^{1}(M, \mathbb{R})$ such that no element of $U$ contains a nowhere vanishing 1 -form;

L2) there is a nonzero class $\xi_{0} \in H^{1}(M, \mathbb{R})$ that contains a nowhere vanishing 1-form.

The existence of such manifolds was indicated in [34, pp. 125-127]. If $M$ has property $(L)$, then the set $\mathcal{V}(M, \rho)$ of all $\rho$-acyclic classes is nonempty for every $\rho$, and by Theorem 5.7 this set equals $H^{1}(M, \mathbb{R}) \backslash\{0\}$ or is the complement in $H^{1}(M, \mathbb{R})$ to a finite union of integral hyperplanes (the case where $\mathcal{V}(M, \rho)=\varnothing$ is excluded by the property L2). Therefore, $\mathcal{V}(M, \rho)$ is open and dense in $H^{1}(M, \mathbb{R})$, and the intersection $I$ of all sets $\mathcal{V}_{\text {alg }}(M, \rho)$ over all right representations in $\operatorname{GL}\left(\mathbf{F}^{n}\right)$ (where $\mathbf{F}$ is a finite field) is a residual subset. Thus, $I$ intersects every open sublet of $H^{1}(M, \mathbb{R})$, in particular, $U \cap I \neq \varnothing$, and therefore there exist cohomology classes $\xi \in H^{1}(M, \mathbb{R})$ that are $\rho$-acyclic for every representation $\rho$ in $\mathrm{GL}\left(\mathbf{F}^{n}\right)$, but not representable by a nowhere vanishing closed 1-form. 
Acknowledgements. A part of the work presented in this paper was done during the author's stay at Osaka City University in December 2003. I was motivated by many discussions with Professors Kunio Murasugi, Akio Kawauchi, Makoto Sakuma, Masaaki Wada, Hiroshi Goda, Teruaki Kitano, Kouji Kodama, Takayuki Morifuji, and Mikami Hirasawa. In particular, the question about the relationship between the Novikov homology and the twisted Alexander polynomial was raised in the course of these discussions. The present paper contains a partial answer to this and other questions of an algebraic nature related to the twisted Novikov homology. I am grateful to Osaka City University and to Professor Akio Kawauchi for the warm hospitality during my stay.

The present paper is a second part of my joint work [11] with Goda. My special thanks go to Hiroshi Goda for sharing his knowledge and insight in knot theory.

Thanks go also to A. Ranicki and Liam O'Carroll for providing useful references on the Fitting invariants of modules.

\section{REFERENCES}

[1] G. Burde and H. Zieschang, Knots, de Gruyter Stud. Math., vol. 5, Walter de Gruyter, Berlin, 1985. MR0808776 (87b:57004)

[2] W. H. Cockcroft and R. G. Swan, On the homotopy type of certain two-dimensional complexes, Proc. London Math. Soc. (3) 11 (1961), 194-202. MR0126271 (23:A3567)

[3] M. M. Cohen, A course in simple-homotopy theory, Grad. Texts in Math., vol. 10, Springer-Verlag, New York-Berlin, 1973. MR0362320 (50:14762)

[4] R. H. Crowell and R. H. Fox, Introduction to knot theory, Ginn and Co., Boston, MA, 1963. MR0146828 (26:4348)

[5] D. Eisenbud, Commutative algebras. With a view toward algebraic geometry, Grad. Texts in Math., vol. 150, Springer-Verlag, New York, 1995. MR1322960 (97a:13001)

[6] M. Farber, Sharpness of Novikov inequalities, Funktsional. Anal. i Prilozhen. 19 (1985), no. 1, 49-59; English transl., Funct. Anal. Appl. 19 (1985), no. 1, 40-48. MR0783706 (86g:58029)

[7] H. Goda, Heegaard splitting for sutured manifolds and Murasugi sum, Osaka J. Math. 29 (1992), 21-40. MR1153177 (93d:57014)

[8] _ On handle number of Seifert surfaces in $S^{3}$, Osaka J. Math. 30 (1993), 63-80. MR1200821 (93k:57010)

[9] H. Goda, T. Kitano, and T. Morifuji, Reidemeister torsion, twisted Alexander polynomial and fibered knots, e-print:math.GT/0311155; Comment. Math. Helv. 80 (2005), no. 1, 51-61. MR2130565 (2005m:57008)

[10] H. Goda and T. Morifuji, Twisted Alexander polynomial for $S L(2, \mathbb{C})$-representations and fibered knots, C. R. Math. Acad. Sci. Soc. R. Can. 25 (2003), no. 4, 97-101. MR2013157 (2004i:57004)

[11] H. Goda and A. Pajitnov, Twisted Novikov homology and circle-valued Morse theory for knots and links, e-print: arXiv.math.GT/0312374; Osaka J. Math. 42 (2005), no. 3, 557-572. MR2166722 (2006e:57015)

[12] B. Jiang and S. Wang, Twisted topological invariants associated with representations, Topics in Knot Theory (Erzurum, 1992), NATO Adv. Sci. Inst. Ser. C Math. Phys. Sci., vol. 399, Kluwer Acad. Publ., Dordrecht, 1993, pp. 211-227. MR1257911

[13] T. Kitano, Twisted Alexander polynomial and Reidemeister torsion, Pacific J. Math. 174 (1996), 431-442. MR1405595 (97g:57007)

[14] F. Latour, Existence de 1-formes fermées non singulières dans une classe de cohomologie de de Rham, Inst. Hautes Études Sci. Publ. Math. No. 80 (1994), 135-194 (1995). MR1320607 (96f:57030)

[15] X. S. Lin, Representations of knot groups and twisted Alexander polynomials, Preprint 1990; Acta Math. Sin. (Engl. Ser.) 17 (2001), 361-380. MR1852950 (2003f:57018)

[16] W. Massey, Homology and cohomology theory, Monogr. Textbooks Pure Appl. Math., vol. 46, Marcel Dekker, Inc., New York-Basel, 1978. MR0488016 (58:7594)

[17] B. R. McDonald, Linear algebra over commutative rings, Monogr. Textbooks Pure Appl. Math., vol. 87, Marcel Dekker, Inc., New York, 1984, 544 pp. MR0769104 (86d:13008)

[18] C. T. McMullen, The Alexander polynomial of a 3-manifold and the Thurston norm on cohomology, Ann. Sci. École Norm. Sup. (4) 35 (2002), no. 2, 153-171; http://abel.math.harvard. edu/ctm/papers. MR1914929 (2003d:57044) 
[19] S. P. Novikov, Multivalued functions and functionals. An analogue of the Morse theory, Dokl. Akad. Nauk SSSR 260 (1981), no. 1, 31-35; English transl., Soviet Math. Dokl. 24 (1981), no. 2, 222-226 (1982). MR0630459 (83a:58025)

[20] - The Hamiltonian formalism and a multivalued analogue of Morse theory, Uspekhi Mat. Nauk 37 (1982), no. 5, 3-49; English transl., Russian Math. Surveys 37 (1982), no. 5, 1-56. MR0676612 (84h:58032)

[21] A. V. Pazhitnov, On the sharpness of inequalities of Novikov type for manifolds with a free abelian fundamental group, Mat. Sb. 180 (1989), no. 11, 1486-1523; English transl., Math. USSR-Sb. 68 (1991), 351-389. MR1034426 (91c:57040)

[22] — Modules over some localizations of the ring of Laurent polynomials, Mat. Zametki 46 (1989), no. 5, 40-49; English transl., Math. Notes 46 (1989), no. 5-6, 856-862 (1990). MR1033419 (91c:13010)

[23] _ On the Novikov complex for rational Morse forms, Preprint, no. 12, Inst. for Mat. og datalogi, Odense Univ., Oct. 1991; Ann. Fac. Sci. Toulouse Math. (6) 4 (1995), 297-338; The pdf-files available at http://www.math.sciences. univ-nantes.fr/pajitnov/. MR1344724 (97f:57033)

[24] - Surgery on the Novikov complex, Rapport de Recherche CNRS URA 758, Nantes, 1993; the pdf-file available at: http://www.math.sciences. univ-nantes.fr/pajitnov/; K-Theory 10 (1996), 323-412. MR1404410 (97j:57043)

[25] — On the asymptotics of Morse numbers of finite covers of manifolds, E-print: math.DG/9810136, 22 Oct 1998; Topology 38 (1999), no. 3, 529-541. MR1670400 (99m:57031)

[26] A. V. Pazhitnov and A. Ranicki, The Whitehead group of the Novikov ring, E-print: math.AT/0012031, 5 dec.2000; K-Theory 21 (2000), no. 4, 325-365. MR1828181 (2002g:19001)

[27] C. Weber, A. Pajitnov, and L. Rudolph (K. Veber, A. Pazhitnov, and L. Rudolf), Morse-Novikov number for knots and links, Algebra i Analiz 13 (2001), no. 3, 105-118; English transl., St. Petersburg Math. J. 13 (2002), no. 3, 417-426. MR1850189 (2002k:57040)

[28] A. Ranicki, The algebraic theory of torsion. I. Foundations, Algebraic and Geometric Topology (New Brunswich, NJ, 1983), Lecture Notes in Math., vol. 1126, Springer, Berlin, 1985, pp. 199-237. MR0802792 (87a:18017)

[29] D. Rolfsen, Knots and links, Math. Lecture Ser., No. 7, Publish or Perish, Inc., Berkeley, CA, 1976; Corrected reprint of the 1976 original, 1990. MR0515288 (58:24236); MR1277811 (95c:57018)

[30] D. Schütz, One-parameter fixed-point theory and gradient flows of closed 1-forms, e-print: math.DG/0104245; K-Theory 25 (2002), 59-97. MR1899700 (2003g:57055)

[31] Gradient flows of closed 1-forms and their closed orbits, e-print: math.DG/0009055; Forum Math. 14 (2002), 509-537. MR1900172 (2003h:57044)

[32] J.-Cl. Sikorav, Points fixes de difféomorphismes symplectiques, intersections de sous-variétés lagrangiennes, et singularités de un-formes fermées, Thése de Doctorat d'Etat Es Sciences Math., Univ. Paris-Sud, Centre d'Orsay, 1987.

[33] Le Ty Kuok Tkhang, Varieties of representations and their subvarieties of cohomology jumps for knot groups, Mat. Sb. 184 (1993), no. 2, 57-82; English transl., Russian Acad. Sci. Sb. Math. 78 (1994), no. 1, 187-209. MR1214944 (94a:57016)

[34] W. Thurston, A norm for the homology of 3-manifolds, Mem. Amer. Math. Soc. 59 (1986), no. 339, 99-130. MR0823443 (88h:57014)

[35] V. Turaev, The Alexander polynomial of a three-dimensional manifold, Mat. Sb. 97 (1975), no. 3, 341-359; English transl., Math. USSR-Sb. 26 (1975), no. 3, 313-329. MR0383425 (52:4306)

[36] _ A norm for the cohomology of 2-complexes, Algebr. Geom. Topol. 2 (2002), 137-155. MR1885218 (2002m:57005)

[37] M. Wada, Twisted Alexander polynomial for finitely presentable groups, Topology 33 (1994), 241256. MR1273784 (95g:57021)

[38] F. Waldhausen, Algebraic K-theory of generalized free products. I, II, Ann. of Math. (2) 108 (1978), 135-204. MR0498807 (58:16845a)

[39] E. Witten, Supersymmetry and Morse theory, J. Differential Geom. 17 (1982), no. 4, 661-692 (1983). MR0683171 (84b:58111)

Laboratoire Mathématiques Jean Leray, UMR 6629, Université de Nantes, Faculté des Sciences, 2, Rue de la Houssinière, 44072, Nantes, Cedex, France

E-mail address: pajitnov@math.univ-nantes.fr

Received 22/FEB/2006

Originally published in English 